\title{
Explicit mixed strain-displacement finite element for dynamic geometrically non-linear solid mechanics
}

\author{
N. M. Lafontaine, R. Rossi, M. Cervera and M. Chiumenti \\ Universidad Politècnica de Catalunya (UPC) \\ Campus Norte UPC, 08034 Barcelona, Spain \\ Centre Internacional de Mètodes Numèrics en Enginyeria (CIMNE) \\ Universidad Politècnica de Catalunya (UPC) \\ Campus Norte UPC, 08034 Barcelona, Spain \\ Web page: http://www.cimne.upc.edu \\ e-mail: maireni@cimne.upc.edu
}

\begin{abstract}
Although appealing for their simplicity, low-order finite elements face inherent limitations related to their poor convergence properties. Such difficulties typically manifest as mesh-dependent or excessively stiff behaviour when dealing with complex problems. A recent proposal to address such limitations is the adoption of mixed displacement-strain technologies which were shown to satisfactorily address both problems. Unfortunately, although appealing, the use of such element technology puts a large burden on the linear algebra, as the solution of larger linear systems is needed. In this paper, the use of an explicit time integration scheme for the solution of the mixed strain-displacement problem is explored as an alternative. An algorithm is devised to allow the effective time integration of the mixed problem. The developed method retains second order accuracy in time and is competitive in terms of computational cost with the standard irreducible formulation.
\end{abstract}

Keywords : explicit, mixed formulation, displacement-strain formulation.

\section{Nomenclature}

$(\bullet):(\bullet)$ Double contraction of tensor (Internal product).

$(\bullet) \cdot(\bullet)$ Single contraction of vector and tensor.

$(\bullet)^{T} \quad$ Vector, matrix transpose.

$(\bullet)_{n} \quad$ Quantity $(\bullet)$ at time $t_{n}$.

$\gamma \quad$ Vector of strain weighting function.

$\boldsymbol{\sigma} \quad$ Stress tensor.

$\varepsilon \quad$ Total strain tensor.

$\boldsymbol{E} \quad$ Green-Lagrange strain tensor.

$\boldsymbol{F} \quad$ Gradient deformation tensor.

$N \quad$ Vector of displacement shape functions.

$\boldsymbol{u} \quad$ Displacement field.

$\boldsymbol{w} \quad$ Vector of displacement weighting function.

$(\bullet)$ Second derivative of $(\bullet)$ with respect to time $t$.

$\Delta t \quad$ Time step. 
$\delta_{i j} \quad$ Kronecker's symbol.

$\mathcal{P}$ Projection operator.

$\mathcal{P}^{\perp} \quad$ Orthogonal projection operator.

$\nabla(\bullet) \quad$ Gradient operator

$\nabla \cdot(\bullet)$ Divergence operator.

$\nabla^{s}(\bullet)$ Symmetric gradient operator $\nabla^{s}(\bullet)=\frac{1}{2}\left(\nabla(\bullet)+\nabla(\bullet)^{T}\right)$.

$\Omega \quad$ A given body without its boundary .

$\partial \Omega \quad \Omega$ 's boundary.

$\rho \quad$ Density material.

$\tau_{\varepsilon} \quad$ Stabilization parameter.

v Poisson's Ratio

$\widetilde{\varepsilon} \quad$ Enhancement strain.

Young's Modulus.

$h^{e} \quad$ Characteristic length of an element.

\section{Tntroduction}

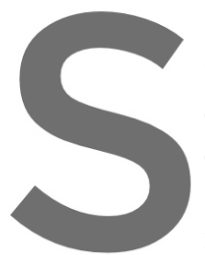

The solution of problems in solid mechanids has be
of the Finite Element Method (FEM), which in tu
otherwise untreatable problems. In structural mech:
and strains are computed as dependent variables
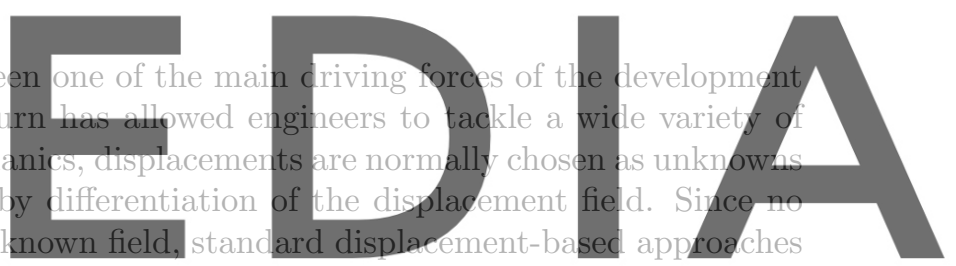

are also known as irreducible formulations. Irreducible formulations are very effective in addressing a

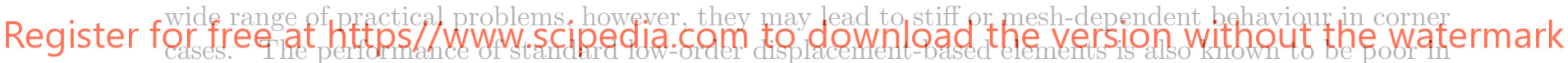

nearly incompressible conditions. Standard low-order elements tend to show "volumetric locking" which

manifests as unrealistically stiff behaviour. A vast literature exists proposing cures for such problems, with early proposals based on reduced integration techniques or on the use of corrected assumed-strains, see for instance, the F-bar method [1]. Mixed displacement-pressure approaches started to be employed in the 90 s for the solution of truly incompressible problems $[1,2,3,4,5]$. Such techniques were also shown to provide an accuracy advantage when used in application to compressible problems (see e.g. [6])

A different mixed formulation is employed here with the goal of improving the accuracy of the Finite Element discretization. The idea is to combine different primary variables, in our case displacements and strains, following the concept described in [7]. This approach was proved to show better mesh independence properties while providing the formal guarantee of a strain field converging at the same rate as the displacement one, which manifests in enhanced stress/analysis accuracy in both linear and non-linear analyses [8]. Following the ideas in [9, 10, 11, 12, 13, 14], a stabilization technique is needed to allow the use of the same order of interpolation for the two primary variables of interest. Specifically the Variational Multiscale Method (VMS) is employed in the current work.

The inherent limitation of the displacement-strain technology is related to its computational cost, since such technique involves higher number of degrees of freedom (dofs) per mesh node (9 vs 3 for the 3D case) which results in much larger linear systems to be solved. The goal of the current paper is to obviate this limitation by exploring the use of an explicit time integration scheme. We will prove that a completely explicit approach is feasible when employing the mixed technology of interest and that it is competitive with the irreducible case. In particular, we will show that, for a given mesh, the critical time 
step is larger for the mixed formulation with respect to the irreducible case, thus enlarging the stability region of the explicit scheme. For this purpose, three different cases are studied. First a simple smalldisplacement example is analysed with the aim of obtaining an analytical estimation of the critical time step. Second, a cantilever beam is studied, again in the small strain regime. Lastly, the same analysis is performed in the large-deformation regime, proving that geometrical nonlinearities can be included within the proposed mixed-displacement formulation, and that such extension comes very natural when an explicit integration scheme is employed.

\section{Mixed Strain/Displacement Formulation In Transient Dy- namics Problems}

\subsection{Continuous problem}

The standard approach in computational solid mechanics is the use of irreducible formulations, in which the deformation is expressed in terms of the derivatives of the displacement field. While this approach is sufficient to satisfactorily address many problems, it suffers from severe limitations when used in combination with low-order finite element discretization. This is evident, for example, in the case of crack-propagation problems, which require guarantee on the local convergence of the strain field, which can not be provided by the standard irreducible element technology. Similar requirements exist however in other areas of application, such as when approaching the incompressible limit.

An appealing possibility to overcome such limitations is the use of mixed formulations, in which additional fields are used as primary variables in combination with the displacement field. Among the many existing possibilities is the use of the two primary variables $u(x)$ and $\varepsilon=\varepsilon(x)$, where $u(x)$ is the displacement field and $\varepsilon(x)$ is a suitable strain measure (e.g the linear strain $\nabla^{s} u$ in small deformations or the Almansi

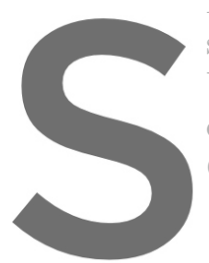
strain in the large defor
by observing that, at co
on $x$ ) coincides with th
Combining this with th
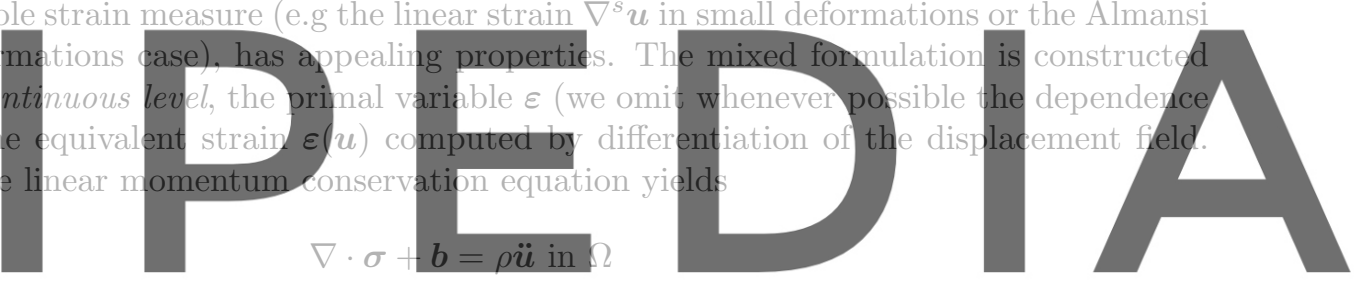

$-\varepsilon(x)+\varepsilon(u)=0$ in $\Omega$

(1)

Register for free at https//www.scipedia.com to download the version without the watermark where $\Omega$ is the domain occupied by the solid in a space of $n_{\text {dim }}$ dimensions, $\rho$ denotes the material density, the superposed dots on $u$ denotes partial differentiation respect to time $t$ (in this case acceleration) and

$\boldsymbol{\sigma}$ is the appropriate stress tensor field, which is generally written as

$$
\sigma=C(\varepsilon): \varepsilon
$$

where $\boldsymbol{C}$ is a fourth-order constitutive tensor, which may include non-linear behaviour. In addition to equations (1), which must hold for all time $t$, the problem is subjected to appropriate Dirichlet and Neumann boundary conditions applied respectively on the portions $\partial \Omega_{\boldsymbol{u}}$ and $\partial \Omega_{\boldsymbol{\sigma}}$ of the boundary, and to the initial conditions expressed by

$$
\begin{aligned}
\left.\dot{\boldsymbol{u}}\right|_{t=0} & =\boldsymbol{v}_{0} \\
\left.\boldsymbol{u}\right|_{t=0} & =\boldsymbol{u}_{0}
\end{aligned}
$$

Introducing equation (2) in equations (1), the strong form becomes

$$
\begin{aligned}
\nabla \cdot(\boldsymbol{C}(\varepsilon): \boldsymbol{\varepsilon})+\boldsymbol{b} & =\rho \ddot{\boldsymbol{u}} \text { in } \Omega \\
-\varepsilon(\boldsymbol{x})+\varepsilon(\boldsymbol{u}) & =\mathbf{0} \quad \text { in } \Omega
\end{aligned}
$$


The two fields $\boldsymbol{u}=\boldsymbol{u}(\boldsymbol{x})$ and $\varepsilon=\varepsilon(\boldsymbol{x})$ can be both discretized by the finite element method (FE) as independent primary variables. Introducing the discrete nodal unknown $\boldsymbol{u}_{h}$ and $\varepsilon_{h}$ and the corresponding test functions $\boldsymbol{w}_{h}$ and $\boldsymbol{\gamma}_{h}$ and applying the Galerkin procedure we thus obtain

$$
\begin{gathered}
\int_{\Omega} \boldsymbol{w}_{h} \cdot\left(\nabla \cdot\left(\boldsymbol{C}\left(\varepsilon_{h}\right): \boldsymbol{\varepsilon}_{h}\right)\right) d \Omega+\int_{\Omega} \boldsymbol{w}_{h} \cdot \boldsymbol{b} d \Omega=\int_{\Omega} \rho \boldsymbol{w}_{h} \cdot \ddot{\boldsymbol{u}}_{h} d \Omega \\
\int_{\Omega} \gamma_{h} \cdot\left(\varepsilon_{h}-\varepsilon\left(\boldsymbol{u}_{h}\right)\right) d \Omega=\mathbf{0}
\end{gathered}
$$

Unfortunately, if equal-order interpolation are used for $\boldsymbol{u}_{h}$ and $\varepsilon_{h}$, the resulting Galerkin method is unstable [15]. Lack of stability manifests as a spurious oscillations in the displacements field that may entirely pollute the solution. A modified formulation needs to be devised to properly stabilize the solution, hence guaranteeing the convergence of the method. Such stabilizations can be derived in the framework of sub-grid approaches [11] as we show next.

\subsection{Stabilized finite element methods}

The origin of the instabilities of the proposed mixed method is that the finite element space employed is not sufficiently large to accommodate the optimal solution. Sub-grid scale stabilization methods retrofit this situation by enlarging the search space, via the introduction of sub-grid variables. The key idea of the sub-grid approach is exactly to consider that the continuous fields can be split in two components, a coarse one, to be solved for, and a finer one to be modelled. Such components correspond to different scales or levels of resolution [7], and are naturally separated in the scales that can be resolved by the finite element discretizations and the ones that can not be resolved at the given discretization level. For the

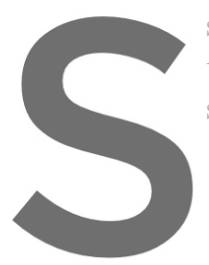
stability of the discrete that is, the effect of the specific problem at han
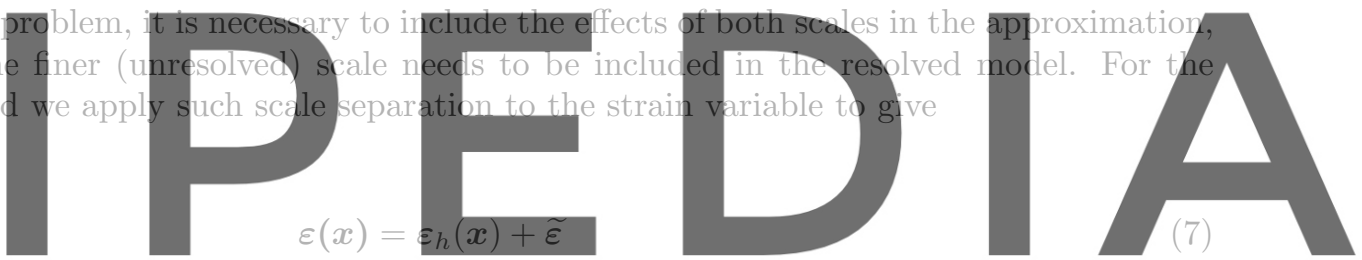

where $\varepsilon_{h}$ is the strain component of the.(coarse) finite element scale and $\widetilde{\varepsilon}$ is the enhancement of the strain

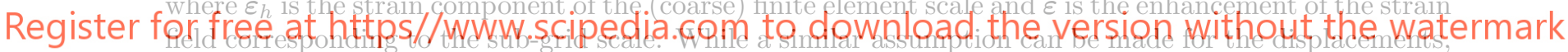
previous experience (see e.g.[7] [8][15]) hints that this splitting is not needed for this variable. Taking into account such considerations, the stabilized model is in the form

$$
\begin{gathered}
\int_{\Omega} \boldsymbol{w}_{h} \cdot\left(\nabla \cdot\left(\boldsymbol{C}\left(\varepsilon_{h}\right):\left(\varepsilon_{h}+\widetilde{\varepsilon}\right)\right)\right) d \Omega+\int_{\Omega} \boldsymbol{w}_{h} \cdot \boldsymbol{b} d \Omega=\int_{\Omega} \rho \boldsymbol{w}_{h} \cdot \ddot{\boldsymbol{u}}_{h} d \Omega \\
\int_{\Omega} \gamma_{h} \cdot\left(\varepsilon_{h}+\widetilde{\varepsilon}-\boldsymbol{\varepsilon}\left(\boldsymbol{u}_{h}\right)\right) d \Omega=\mathbf{0}
\end{gathered}
$$

substituting $\boldsymbol{C}\left(\varepsilon_{h}\right)$ instead of $\boldsymbol{C}\left(\varepsilon_{h}+\widetilde{\boldsymbol{\varepsilon}}\right.$ ) (see [7] [8] [15]). Next a model needs to be chosen for $\widetilde{\boldsymbol{\varepsilon}}$. Following the idea of Orthogonal Subscale Stabilization (OSS), we assume that the subgrid strain is proportional to the orthogonal projection of the coarse scale residual, that is, the strain residual $\left(\boldsymbol{r}_{\varepsilon}:=\varepsilon\left(\boldsymbol{u}_{h}\right)-\varepsilon_{h}\right)$ is introduced, and

$$
\widetilde{\varepsilon}=\tau_{\epsilon} \mathcal{P}^{\perp}\left(\boldsymbol{r}_{\varepsilon}\right)
$$

here $\mathcal{P}^{\perp}$ indicates the orthogonal projection operation (such that $\mathcal{P}^{\perp}(\bullet)=\mathcal{J}-\mathcal{P}(\bullet)$ and $\mathcal{P}^{\perp}\left(\varepsilon_{h}\right)=\mathbf{0}$ ) and $\tau_{\epsilon}$ is the algorithmic stabilization parameter, theoretically defined as

$$
\tau_{\epsilon}=c_{\varepsilon} \frac{h}{L}
$$


with $c_{\varepsilon}, h$ and $L$ being an "arbitrary" algorithmic constant, the element size and the characteristic length of the computational domain respectively. For simplicity, in the case of constant meshes it is customary to take a constant value of the stabilization parameter[8][15]. In this work typical values, in the range 0.1-0.5, were considered in all the examples.

Taking into account such definition, equation (10) gives

$$
\begin{aligned}
\widetilde{\varepsilon} & =\tau_{\epsilon}\left(\boldsymbol{r}_{\boldsymbol{\varepsilon}}-\mathcal{P}\left(\boldsymbol{r}_{\varepsilon}\right)\right) \\
& =\tau_{\epsilon}\left(\boldsymbol{\varepsilon}\left(\boldsymbol{u}_{h}\right)-\boldsymbol{\varepsilon}_{h}-\mathcal{P}\left(\varepsilon\left(\boldsymbol{u}_{h}\right)\right)+\mathcal{P}\left(\varepsilon_{h}\right)\right) \\
& =\tau_{\epsilon}\left(\boldsymbol{\varepsilon}\left(\boldsymbol{u}_{h}\right)-\mathcal{P}\left(\varepsilon\left(\boldsymbol{u}_{h}\right)\right)\right)
\end{aligned}
$$

Replacing into equation (6a) and simplifying we obtain

$$
\begin{gathered}
\boldsymbol{\varepsilon}_{h}=\mathcal{P}\left(\boldsymbol{\varepsilon}\left(\boldsymbol{u}_{h}\right)\right) \\
\boldsymbol{\varepsilon}^{s t a b}=\left(1-\tau_{\epsilon}\right) \boldsymbol{\varepsilon}_{h}+\tau_{\epsilon} \boldsymbol{\varepsilon}\left(\boldsymbol{u}_{h}\right) \\
\int_{\Omega} \rho w_{h} \cdot \ddot{u}_{h} d \Omega+\int_{\Omega} \nabla^{s} w_{h}:\left(C\left(\varepsilon_{h}\right):\left(\varepsilon^{s t a b}\right)\right) d \Omega=\int_{\Omega} w_{h} \cdot b_{h} d \Omega+\int_{\partial \Omega} w_{h} \cdot t_{h} d \Gamma
\end{gathered}
$$

which define the stable discrete problem to be solved. Note that the term added in equation (13b) to secure a stable solution decreases rapidly upon mesh refinement, since $\left\|\varepsilon\left(\boldsymbol{u}_{\boldsymbol{h}}\right)-\varepsilon\right\| \rightarrow 0$.

\subsubsection{Remark: Projection Operations}

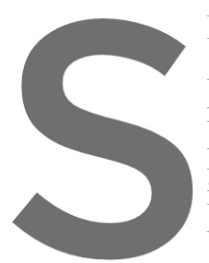

It is interesting to make

A projector $\mathcal{P}$ is a linear

representation of such $v$

property $\mathcal{P}(y)=\mathcal{P}(\mathcal{P}(y)$

itself. A non-orthogonal pr

that is, $\boldsymbol{y}_{h}=\mathcal{P}(y)$.
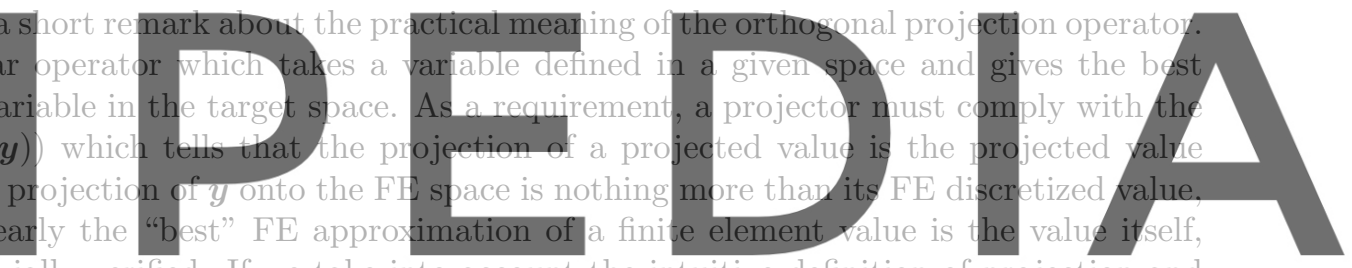

hence $y_{h}=\mathcal{P}\left(y_{h}\right)$ is trivially verified. If we take into account the intuitive definition of projection and

we take as "best" a least-square approximation, we can readily see that the projected value $y_{h}$ can be

Register fortfree at https//www.scipedia.com to download the version without the watermark

$$
\int_{\Omega} \boldsymbol{w}_{h} \cdot\left(\boldsymbol{y}_{h}-\boldsymbol{y}(\boldsymbol{x})\right) d \Omega=\mathbf{0}
$$

which gives rise to a discrete problem of the type

$$
\int_{\Omega} \boldsymbol{w}_{h} \cdot \boldsymbol{y}_{h} d \Omega=\int_{\Omega} \boldsymbol{w}_{h} \cdot \boldsymbol{y}(\boldsymbol{x}) d \Omega
$$

we recognize on the left side a mass-like operator $\breve{M}$, so that such operation can be understood as

$$
\breve{\boldsymbol{M}} \boldsymbol{y}_{h}=\int_{\Omega} \boldsymbol{w}_{h} \cdot \boldsymbol{y}(\boldsymbol{x}) d \Omega
$$

If mass lumping techniques are applied to $\breve{M}$ a diagonal matrix is defined with each diagonal term representing the nodal area of the corresponding node of the FE mesh. Under such assumption, the projection operator can be approximated as an area-weighted smoothing of the original variable. By definition, the orthogonal projection of the variable is the part of the variable that is orthogonal to the FE space, that is, the part that can not be represented onto this space. We can define constructively the orthogonal projector as the operator that takes away from the projected variable everything that can be represented in the FE space. Since the projection operator is linear, this is done as

$$
\mathcal{P}^{\perp}(\boldsymbol{y}):=\boldsymbol{y}-\mathcal{P}(\boldsymbol{y})=\boldsymbol{y}-\boldsymbol{y}_{h}
$$


with this definition is immediately verified that

$$
\mathcal{P}^{\perp} \mathcal{P}\left(\boldsymbol{y}_{h}\right)=\mathcal{P}\left(\boldsymbol{y}_{h}\right)-\mathcal{P}\left(\mathcal{P}\left(\boldsymbol{y}_{h}\right)\right)=\boldsymbol{y}_{h}-\boldsymbol{y}_{h}=\mathbf{0}
$$

thus proving the orthogonality.

\section{Large deformation case}

To handle large deformations, the strain $\varepsilon(\mathbf{u})$ needs to be defined as a large strain measure. A natural possibility is to define it as the Almansi strain as $\varepsilon(\mathbf{u}):=\frac{1}{2}\left(\nabla \mathbf{u}+\nabla^{t} \mathbf{u}+\nabla \mathbf{u} \nabla^{t} \mathbf{u}\right)$ where the the gradients are computed with respect to the deformed configuration. The discrete system can then be obtained by integrating equation (9) onto the current (deformed) configuration $\Omega_{t}$, as normally done in Updated Lagrangian approaches.

An equivalent formulation can be obtained by integrating on the reference (undeformed) domain $\Omega_{0}$, provided that appropriate conjugate strain measures are employed. The equivalent Total Lagrangian formulation can be obtained following the traditional procedure described e.g. in [16]. We followed here this approach in writing our system.

Introducing the Cauchy-Green strain (CG) measure

$$
\boldsymbol{E}=\frac{1}{2}\left(\boldsymbol{F}^{T} \boldsymbol{F}-\boldsymbol{I}\right)
$$

and a properly defined elasticity tensor $C$ we can compute the second Piola-Kirchhoff (PK2) stress $S=C(\mathbb{E}, S): E$ whic
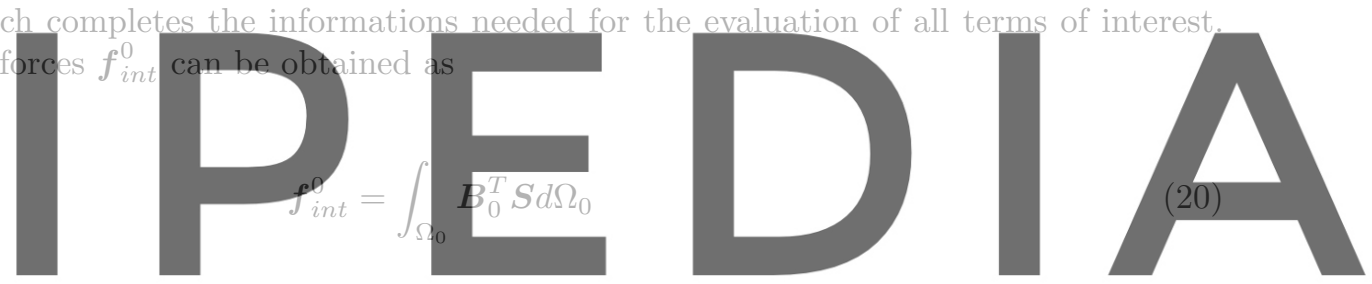

where, $F:=\frac{\partial x}{}$ is the deformation gradient. Strain variations can be computed on the basis of the Register for free at a

Matrix $B_{0}$ (used in the Total Lagrangian approach) is composed of submatrices, each associated with a node $I$. For a particular node $I$, the sub-matrix $\boldsymbol{B}_{0}^{I}$ is written in Voigt form as:

$$
\boldsymbol{B}_{0}^{I}=\left(\begin{array}{cc}
\frac{\partial N_{I}}{\partial X} \frac{\partial x}{\partial X} & \frac{\partial N_{I}}{\partial X} \frac{\partial y}{\partial X} \\
\frac{\partial N_{I}}{\partial y} \frac{\partial x}{\partial Y} & \frac{\partial N_{I}}{\partial Y} \frac{\partial y}{\partial Y} \\
\frac{\partial N_{I}}{\partial X} \frac{\partial x}{\partial Y}+\frac{\partial N_{I}}{\partial Y} \frac{\partial x}{\partial X} & \frac{\partial N_{I}}{\partial X} \frac{\partial y}{\partial Y}+\frac{\partial N_{I}}{\partial Y} \frac{\partial y}{\partial X}
\end{array}\right)
$$

For the Updated Lagrangian case, the corresponding matrix is $\boldsymbol{B}_{t}$ :

$$
\boldsymbol{B}_{t}^{I}=\left(\begin{array}{cc}
\frac{\partial N_{I}}{\partial x} & 0 \\
0 & \frac{\partial N_{I}}{\partial y} \\
\frac{\partial N_{I}}{\partial y} & \frac{\partial N_{I}}{\partial x}
\end{array}\right)
$$

Table 1 summarizes the values of the corresponding terms in Total Lagrangian and Updated Lagrangian approaches. 


\begin{tabular}{|c|c|c|}
\hline \multirow[b]{2}{*}{ Entity } & \multicolumn{2}{|c|}{ Integration Domain $\Omega$} \\
\hline & Total Lagrangian $\Omega_{0}$ & Updated Lagrangian $\Omega_{t}$ \\
\hline Strain & $(\mathrm{CG}) \boldsymbol{E}\left(\boldsymbol{u}_{h}\right):=\frac{1}{2}\left(\boldsymbol{F}^{T} \boldsymbol{F}-\boldsymbol{I}\right)$ & $(\mathrm{AL}) \boldsymbol{e}\left(\mathbf{u}_{h}\right):=\frac{1}{2}\left(\boldsymbol{I}-\boldsymbol{F}^{-T} \boldsymbol{F}^{-1}\right)$ \\
\hline Projection & $\int_{\Omega_{0}} \gamma_{h} \cdot\left(\boldsymbol{E}_{h}-\boldsymbol{E}\left(\boldsymbol{u}_{h}\right)\right) d \Omega_{0}=\mathbf{0}$ & $\int_{\Omega} \gamma_{h} \cdot\left(\boldsymbol{e}_{h}-\boldsymbol{e}\left(\boldsymbol{u}_{h}\right)\right) d \Omega=\mathbf{0}$ \\
\hline Stabilized Strain & $\boldsymbol{E}^{\text {stab }}=\left(1-\tau_{\epsilon}\right) \boldsymbol{E}_{h}+\boldsymbol{E}\left(\boldsymbol{u}_{h}\right)$ & $\boldsymbol{e}^{s t a b}=\left(1-\tau_{\epsilon}\right) \boldsymbol{e}_{h}+\boldsymbol{e}\left(\boldsymbol{u}_{h}\right)$ \\
\hline Stress(irreducible) & $(\mathrm{PK} 2) \boldsymbol{S}=\boldsymbol{C}_{0}: \boldsymbol{E}$ & Cauchy $\sigma=C: e$ \\
\hline Stress(mixed) & $(\mathrm{PK} 2) \boldsymbol{S}^{\text {stab }}=\boldsymbol{C}_{0}: \boldsymbol{E}^{\text {stab }}$ & Cauchy $\sigma^{s t a b}=C: e^{s t a b}$ \\
\hline External Forces & $\boldsymbol{f}_{0}^{e x t}=\int_{\partial \Omega_{0}} \boldsymbol{W}_{h} \cdot \boldsymbol{T} d \Gamma_{0}$ & $\boldsymbol{f}^{e x t}=\int_{\partial \Omega} \boldsymbol{w}_{h} \cdot \boldsymbol{t} d \Gamma$ \\
\hline Internal Forces(irreducible) & $\boldsymbol{f}_{0}^{i n t}=\int_{\Omega_{0}} \boldsymbol{B}_{0}^{T} \boldsymbol{S} d \Omega_{0}$ (Voigt Notation) & $\boldsymbol{f}^{i n t}=\int_{\Omega} \boldsymbol{B}_{t}^{T} \boldsymbol{\sigma} d \Omega($ Voigt Notation $)$ \\
\hline Internal Forces(mixed) & $\boldsymbol{f}_{0}^{\text {int }}=\int_{\Omega_{0}} \boldsymbol{B}_{0}^{T} \boldsymbol{S}^{\text {stab }} d \Omega_{0}$ (Voigt Notation) & $\boldsymbol{f}^{i n t}=\int_{\Omega} \boldsymbol{B}_{t}^{T} \boldsymbol{\sigma}^{s t a b} d \Omega$ (Voigt Notation) \\
\hline
\end{tabular}

Table 1: Entities measures in both reference $\Omega_{0}$ and deformed $\Omega_{t}$ configuration for irreducible and mixed formulation.

As a final remark, we would like to observe how, in the Total Lagrangian case, it is natural to express the projection operator in terms of $\boldsymbol{E}$ as

$$
\int_{\Omega_{0}} \gamma_{h} \cdot\left(\boldsymbol{E}_{h}-\boldsymbol{E}\left(\boldsymbol{u}_{h}\right)\right) d \Omega_{0}=0
$$

This choice is not exactly equivalent to projecting the Almansi strain (AL) $e$ in the deformed domain, but rather corresponds to a slightly different definition of the projection operator. In other words, the

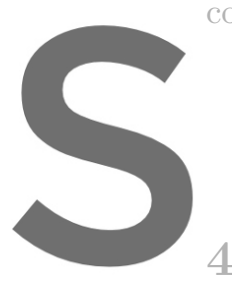
corresponding projection
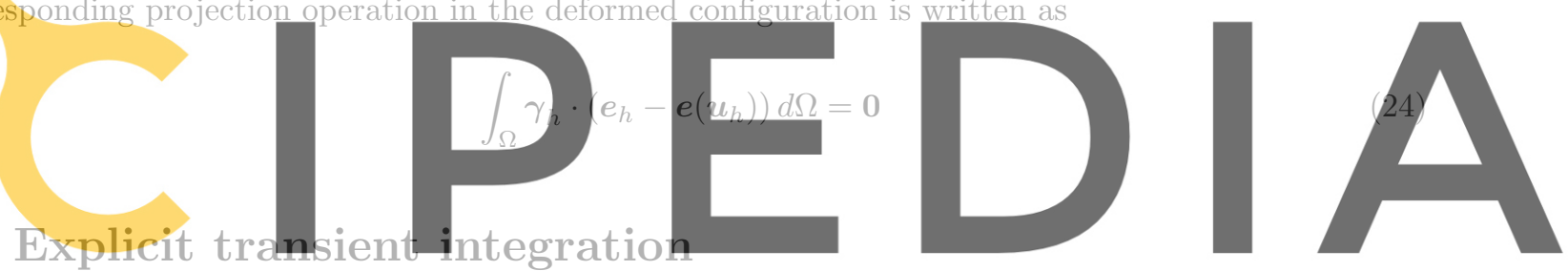

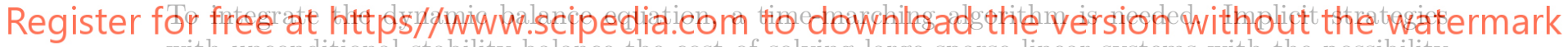
with unconditional stability balance the cost of solving large sparse linear systems with the possibility of using large time steps. Explicit solvers are usually considered advantageous in addressing very short transient phenomena or very high non-linearities need to be faced.

For the case of the mixed displacement-strain formulation no time derivative is present for the strains, making less straightforward the definition of an explicit technique. The aim of the next section is to propose a feasible, completely explicit, algorithm for the time integration. Within the same section we also prove that the stability limit for the proposed explicit algorithm is not worse (and generally better) than for the corresponding irreducible formulation. To do this we first recall the standard time integration procedure, and then we develop the integration procedure applied to the mixed formulation described herein.

\subsection{Standard formulation in explicit dynamics (irreducible problem)}

A commonly used algorithm in the time integration of problems in structural dynamics is the Central Differences $(\mathrm{CD})$ time marching scheme. As shown in figure (1), the essential idea of the CD is that the dynamic equilibrium is written at time $n$ so to allow the evaluation of the mid-step velocity and successively of the displacements.

If we consider the discrete dynamic equilibrium problem in the form

$$
\boldsymbol{M} \ddot{\boldsymbol{u}}_{h}(t)+\boldsymbol{D} \dot{\boldsymbol{u}}_{h}(t)+\boldsymbol{f}^{i n t}\left(\boldsymbol{u}_{h}, t\right)=\boldsymbol{f}^{\text {ext }}(t)
$$




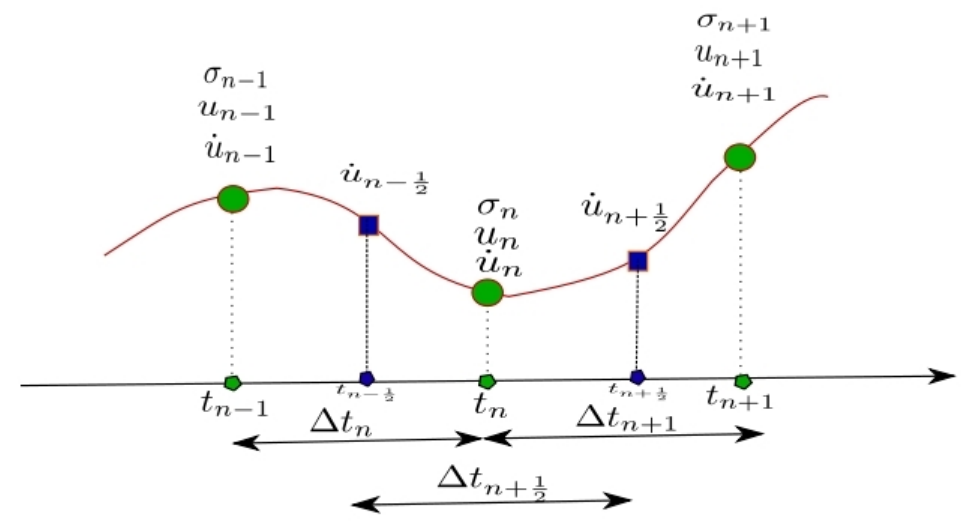

Figure 1: Central Differences Scheme

where $M$ and $D$ are the mass and damping matrices, assumed diagonal, $f^{i n t}(u)$ and $f^{e x t}$ are the nodal vector of internal and external forces, and $\ddot{u}, \dot{u}$ and $u$ are the accelerations, velocities and displacements respectively.

Assuming at time step $\Delta t$, the straightforward application of the CD to equation(25) leads to the procedure
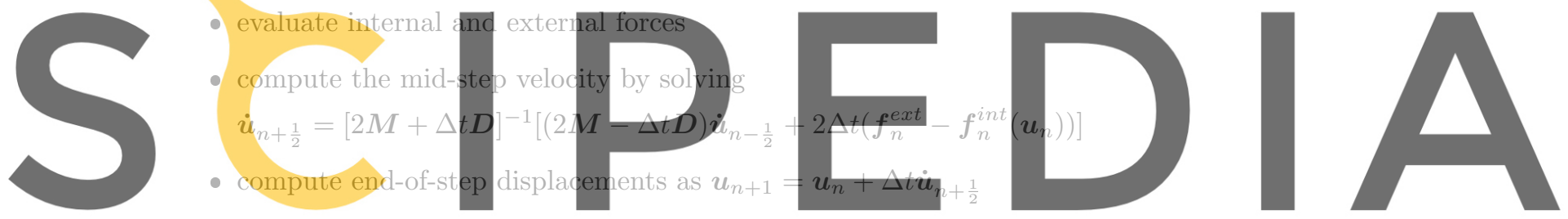

where subscripts $n$ and $n+1$ are used to indicate quantities at time $t=t_{n}$ or $t=t_{n+1}$. Note that the

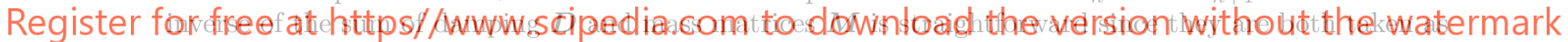

diagonal. The critical time step is given by the Courant limit, which is the time necessary for a sound wave to cross the smallest element in the mesh[17]. For the undamped case this critical time step is given by

$$
\Delta t_{c r} \leq \frac{2}{\omega_{\max }}
$$

where $\omega_{\max }$ is the maximum eigenvalue associated to the system's stiffness matrix. The frequencies of the discrete system are bounded by the maximum frequency, $\omega_{\max }^{e}$ of the individual elements. A useful approximation is given by.

$$
\omega_{\text {max }}^{e} \approx \frac{2 c^{e}}{l_{\text {min }}^{e}} ; l_{\text {min }}^{e}=\min _{e}\left\{h^{e}\right\}
$$

where $c^{e}$ is the elastic dilational wave speed and $l_{\text {min }}^{e}$ is the smallest characteristic element size in the finite element mesh corresponding to the smallest distance between any two element nodes in the discretized model. Therefore, the critical time is finally expressed as:

$$
\Delta t_{c r} \approx \frac{l_{\min }^{e}}{c^{e}}
$$




\subsection{Mixed formulation in explicit dynamics}

If we now focus on the mixed strain-displacement formulation, the discrete equation of motion (25) is replaced by

$$
\boldsymbol{M} \ddot{\boldsymbol{u}}_{h}(t)+\boldsymbol{D} \dot{\boldsymbol{u}}_{h}(t)+\boldsymbol{f}^{i n t}\left(\varepsilon^{s t a b}, t\right)=\boldsymbol{f}^{e x t}(t)
$$

which differs from equation $(25)$ in that the internal forces depend on $\varepsilon^{s t a b}$, i.e

$$
\boldsymbol{f}^{i n t}\left(\varepsilon^{s t a b}\right)=\int_{\Omega} \boldsymbol{B}^{T} \boldsymbol{\sigma} d \Omega=\int_{\Omega} \boldsymbol{B}^{T} \boldsymbol{C}: \boldsymbol{\varepsilon}^{s t a b} d \Omega
$$

Taking into account the definition of $\varepsilon^{\text {stab }}$ given in equation (13b) and replacing it into equation (30), equation (29) can be rewritten as a set of two equations:

$$
\begin{aligned}
M \ddot{u}_{h}(t)+D \dot{u}_{h}(t)+\overbrace{\bar{K}_{\tau} u_{h}+G_{\tau}^{T} \varepsilon_{h}}^{f^{i n t}(t)} & =f^{e x t}(t) \\
\breve{G} u_{h}-\breve{M} \varepsilon_{h} & =0
\end{aligned}
$$

where (31a) represents the modified dynamic equilibrium and (31b) tells that $\varepsilon_{h}$ shall be the projection of $\varepsilon(u)$. In equations (31b), $\breve{M}$ is a diagonal mass matrix associated to the strain field (needed for the computation of the orthogonal projection of the strain variable); $G_{\tau}$ and $G$ are the discrete symmetric gradient operators and $\boldsymbol{K}$ element, these matrices can be writt
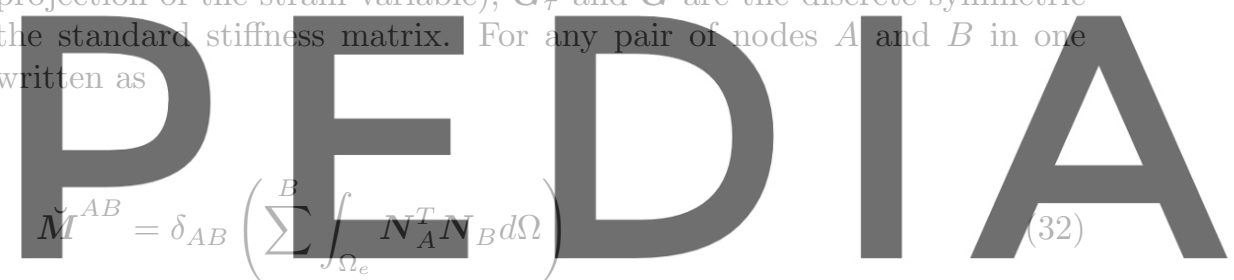

Register for free at https//www.scipedia.com to download the version without the watermark

$$
\begin{gathered}
G_{\tau}^{A B}=\left(1-\tau_{\epsilon}\right) \int_{\Omega_{e}} B_{A}^{T} C\left(\varepsilon_{h B}\right) N_{B} d \Omega \\
\breve{\boldsymbol{G}}^{A B}=\int_{\Omega_{e}} \boldsymbol{B}_{A}^{T} \boldsymbol{N}_{B} d \Omega \\
\boldsymbol{K}_{\tau}^{A B}=\tau_{\epsilon} \int_{\Omega_{e}} \boldsymbol{B}_{A}^{T} \boldsymbol{C}\left(\boldsymbol{\varepsilon}_{h}\right) \boldsymbol{B}_{B} d \Omega
\end{gathered}
$$

where $\delta_{A B}$ is the Kronecker delta $\left(\delta_{A B}=1\right.$ for $A=B, \delta_{A B}=0$ if $\left.A \neq B\right)$.

It is convenient to rewrite equations (31a) and (31b) as

$$
\left(\begin{array}{cc}
\boldsymbol{M} & \mathbf{0} \\
\mathbf{0} & \mathbf{0}
\end{array}\right)\left(\begin{array}{l}
\ddot{\boldsymbol{u}} \\
\mathbf{0}
\end{array}\right)+\left(\begin{array}{cc}
\boldsymbol{D} & \mathbf{0} \\
\mathbf{0} & \mathbf{0}
\end{array}\right)\left(\begin{array}{c}
\dot{\boldsymbol{u}} \\
\mathbf{0}
\end{array}\right)+\left(\begin{array}{cc}
\boldsymbol{K}_{\tau} & \boldsymbol{G}_{\tau}^{T} \\
\breve{G} & -\breve{M}
\end{array}\right)\left(\begin{array}{c}
\boldsymbol{u} \\
\varepsilon_{\boldsymbol{h}}
\end{array}\right)=\left(\begin{array}{c}
\boldsymbol{f}^{\text {ext }} \\
\mathbf{0}
\end{array}\right)
$$

Since this form will make easier eigenvalue computations in the calculation of the critical time step. For this reason we introduce the matrices

$$
\boldsymbol{K}_{\text {mixed }}:=\left(\begin{array}{cc}
\boldsymbol{K}_{\tau} & \boldsymbol{G}_{\tau}^{T} \\
\breve{G} & -\breve{M}
\end{array}\right)
$$




$$
M_{\text {mixed }}:=\left(\begin{array}{cc}
M & 0 \\
\mathbf{0} & 0
\end{array}\right)
$$

which we will use in the next section. Written in this form, the discrete system is very similar to the one developed for the irreducible case, except that two independent unknowns variables, i.e, the nodal displacement field $\boldsymbol{u}_{\boldsymbol{h}}$ and the nodal strain field $\varepsilon_{h}$ are now considered. We can however observe that since $\breve{M}$ is diagonal and equation (31b) does not present any time dependence, we can formally express the continuous strain as a function of the displacements field as, $\boldsymbol{\varepsilon}_{\boldsymbol{h}}=\breve{\boldsymbol{M}}_{\tau}^{-1} \breve{\boldsymbol{G}} \boldsymbol{u}$ and substitute it into the equilibrium equation (31a). By introducing the new symbol

$$
\overline{\boldsymbol{K}}:=\boldsymbol{K}_{\tau}+\boldsymbol{G}_{\tau}^{T} \breve{M}_{\tau}^{-1} \breve{G}
$$

we can thus rewrite equation (31a) in terms of displacements only in the equivalent form

$$
M \ddot{u}_{h}(t)+D \dot{u}_{h}(t)+\bar{K} u_{h}=f^{e x t}(t)
$$

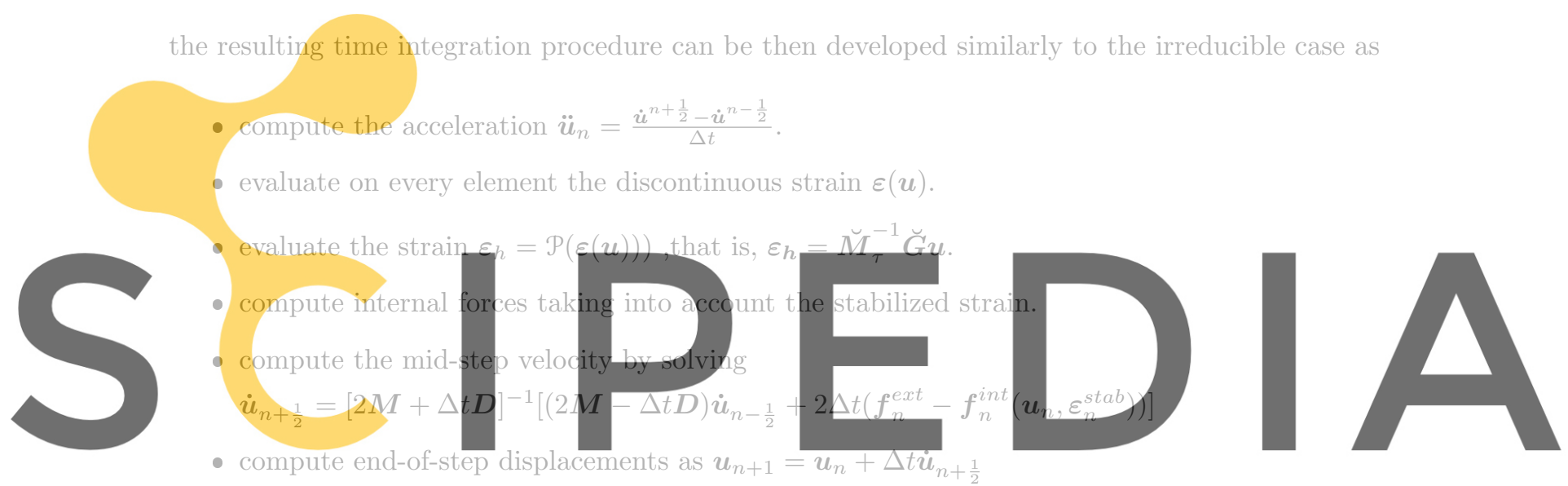

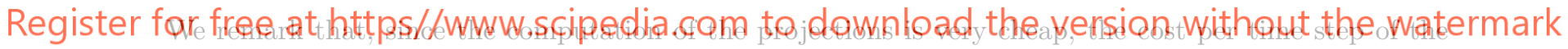
proposed scheme is very similar to that of the original irreducible algorithm, making it very interesting in view of the accuracy advantage guaranteed by the mixed formulation. In the next subsection we discuss the critical time step for the proposed algorithm, considering first $1 D$ case, for which an analytical estimate is obtained. We then consider a $2 D$ mesh and provide a numerical verification of the formula.

Before proceeding we would also like to point out that the form used in writing the discrete system obtained is slightly different from the one employed in [7], in that in the current approach the $\breve{M}$ is purely diagonal. The approach we follow stems out of using a nodal integration rule in the calculation of the projection and by noticing that the resulting mass term is block diagonal and can thus be solved analytically block by block to make it diagonal. We also observe that this approach is not appealing for the implicit case since it results in a non-symmetric matrix.

\subsection{Stability of the explicit mixed formulation}

\subsubsection{D mixed bar problem}

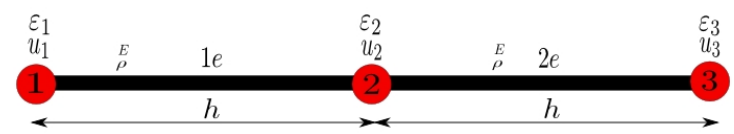

Figure 2: one-dimensional bar example. 
Let us consider the one-dimensional problem in figure (2). This corresponds to a bar with unit cross section, discretized using two elements of length $h$. Constant material properties Young's modulus $E$ and density $\rho$ are assumed. The degrees of freedom of displacement $u_{1}$ and $u_{3}$ are fixed for the nodes 1 and 3. For irreducible case, the system's fundamental frequency is given by:

$$
\omega_{\text {irre }}=\sqrt{\frac{2 E}{\rho h^{2}}}
$$

It is possible to compute analytically the eigenvalues of the mixed problem by evaluating the eigenvalues of the corresponding discrete system. Here $A$ and $B$ represent the local nodes corresponding to the two ends of a 1D bar element. The same linear interpolation function $\boldsymbol{N}$ are used for the strain and displacement fields. Applying the mixed formulation, the local mixed stiffness matrix $\boldsymbol{K}_{\text {mixed }}^{A B}$ of one bar element is a $4 \times 4$ matrix compose by terms affecting degrees of freedom of displacements and strains. The sub-matrices $\breve{M}, \breve{G}, \boldsymbol{G}_{\tau}$ and $\boldsymbol{K}_{\tau}$ defined in (32-35) for one mixed bar element are

$$
\begin{gathered}
\breve{M}^{A B}=\frac{h}{2}\left(\begin{array}{cc}
1 & 0 \\
0 & 1
\end{array}\right) \\
\boldsymbol{K}_{\tau}^{A B}=\tau_{\epsilon} \frac{E}{h}\left(\begin{array}{cc}
1 & -1 \\
-1 & 1
\end{array}\right) \\
\breve{G}^{A B}=\frac{1}{2}\left(\begin{array}{cc}
-1 & -1 \\
1 & 1
\end{array}\right) \\
\boldsymbol{G}_{\tau}^{A B}=\frac{E\left(1-\tau_{\epsilon}\right)}{2}\left(\begin{array}{cc}
-1 & -1 \\
1 & 1
\end{array}\right)
\end{gathered}
$$

The mass matrix for each mixed bar element is trivially computed as

$$
M^{A B}=\frac{\rho h}{2}\left(\begin{array}{ll}
1 & 0 \\
0 & 1
\end{array}\right)
$$

Accordingly, the local mixed stiffness matrix $\boldsymbol{K}_{\text {mixed }}$ of a bar element is the assembly of these matrices. Hence, using the expression in equation (37), it can finally express the mixed local stiffness matrix of a bar element

$$
\boldsymbol{K}_{\text {mixed }}^{A B}=\left(\begin{array}{cccc}
\frac{E \tau_{\epsilon}}{h} & \frac{-E \tau_{\epsilon}}{h} & \frac{E\left(\tau_{\epsilon}-1\right)}{2} & -\frac{E \tau_{\epsilon}}{2} \\
\frac{-E \tau_{\epsilon}}{h} & \frac{E \tau_{\epsilon}}{h} & \frac{E\left(\tau_{\epsilon}-1\right)}{2} & -\frac{E\left(\tau_{\epsilon}-1\right)}{2} \\
-\frac{1}{2} & -\frac{1}{2} & -\frac{h}{2} & 0 \\
\frac{1}{2} & \frac{1}{2} & 0 & -\frac{h}{2}
\end{array}\right)
$$

Assembling the elemental contribution of both $\boldsymbol{K}_{\text {mixed }}$ and $\boldsymbol{M}_{\text {mixed }}$ and fixing the displacements of node 1 and 3 , we can obtain the relevant natural frequencies by solving

$$
\begin{aligned}
\operatorname{det}\left(\boldsymbol{K}_{\text {mixed }}-\lambda \boldsymbol{M}_{\text {mixed }}\right) & =0 \\
\omega_{\text {mixed }}^{2} & =\lambda
\end{aligned}
$$




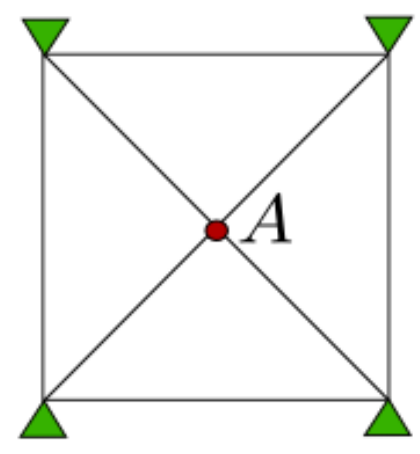

(a) $2 D$ case test for stability analysis order time accuracy.

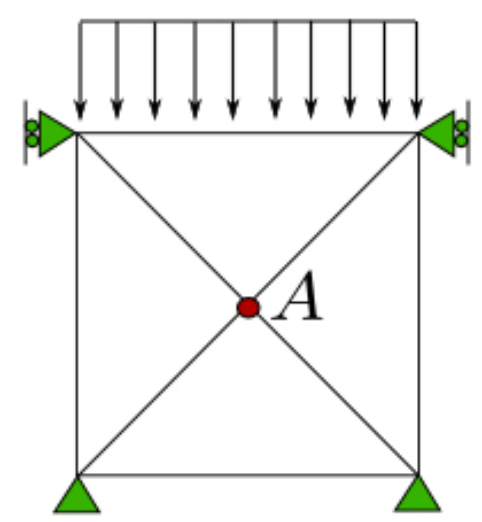

(b) $2 D$ case test for checking second

Figure 3: $2 D$ case test.

Therefore, after some algebra we obtain the following analytical expression for the first frequency of the mixed system:

$$
\omega_{\text {mixed }}=\sqrt{\frac{E\left(1+\tau_{\epsilon}\right)}{\rho h^{2}}}
$$

As expected the irreducible solution (see equation(41)) can be recovered for $\tau_{\epsilon}=1$ (which is the limit case at which only the discontinuous strain is taken into account).

\subsection{2 $2 D$ case}

Consider now a $2 D$ benchmark example, described in figure (3a) to verify numerically that the $1 D$ predictions also apply to the $2 D$ case. The model has an unit dimension $1 \times 1$ and is discretized in four elements. The corner nodes are fixed. Density $\rho$, Young's Modulus $E$, thickness $t$ and Poisson ratio $\nu$ are $100 \mathrm{~kg}, 10^{6} \mathrm{~N} / \mathrm{m}^{2}, 1 \mathrm{~m}$ and 0.3 respectively. As the above example, a modal analysis is performed for irreducible and mixed formulation.

The plot of $\omega_{\text {mixed }} / \omega_{\text {irre }}$, for varying values of the stabilization parameter is given in figure (4). $\omega$ represents here the highest frequency in the system. As predicted by the theoretical analysis, the system becomes more and more flexible as the stabilization parameter decreases, to reach a minimal frequency of about half that of the original irreducible model when the stabilization parameter approaches zero. The immediate implication is that the critical time step for the explicit dynamic analysis is greater for the mixed formulation than for the irreducible one. The numerical experiments shown in figure (5) show that, the estimated gain for the two-dimensional case is even more favourable than for the one-dimensional case.

The simple mesh considered here is also suitable to verify that second order time accuracy is retained by the modified algorithm. To verify this fact, we slightly modify the boundary conditions and consider the settings shown in figure (3b). A dynamic analysis is performed, comparing the mixed and the irreducible formulation. Since a Lagrangian approach is followed, the spatial error and the time integration error are decoupled. We can hence compute a reference solution employing an extremely small time step. We then compute the solution using different time steps and sample the values of displacement and strain at the point $A$ of figure (3b) at the time instant $t=0.4 s$. By comparing the results to the reference we can then plot (in logarithmic scale) the error as a function of the employed time step size. Since the curve $\Delta t^{2}$ shown in figure(6) is parallel to the error plot, the graph proves the expected convergence rates. 


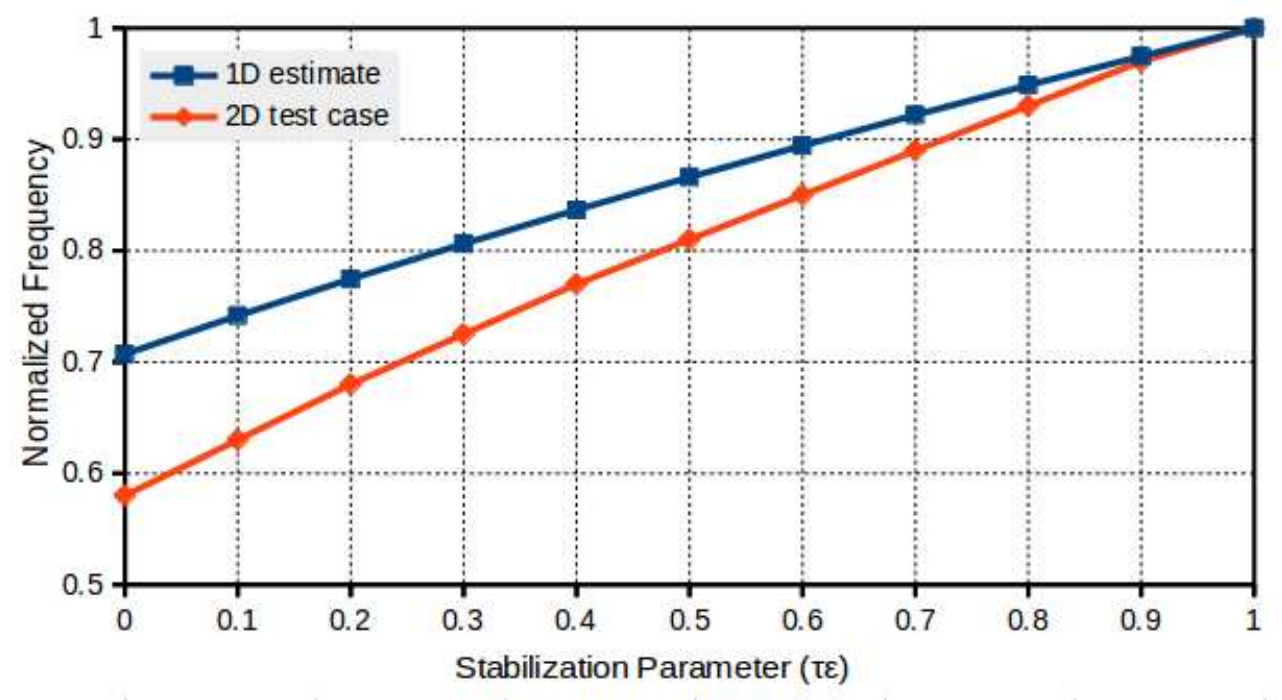

Figure 4: Variation of normalized frequency with stabilization parameter.

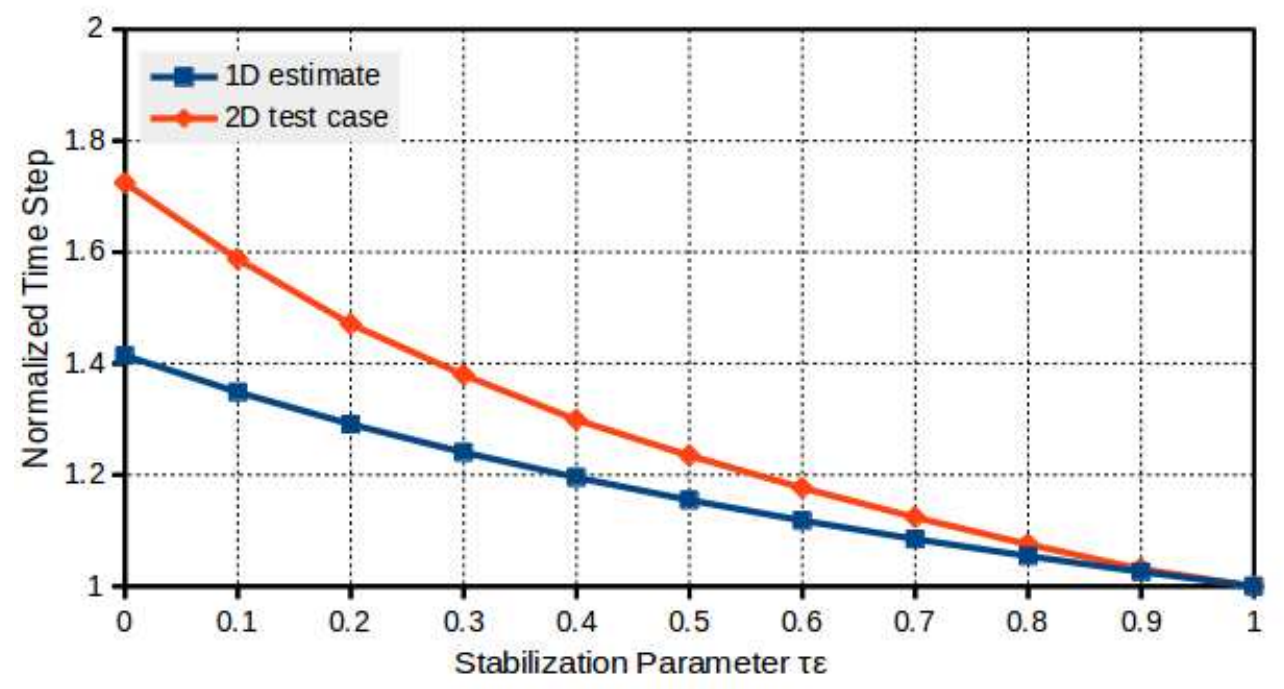

Figure 5: Variation of normalized Time with stabilization parameter.

\subsection{Numerical accuracy of the mixed formulation}

In this section the accuracy of the proposed mixed explicit formulation is assessed in the application to a real transient dynamic problem, in both small and large deformations. The test case consists of a cantilever beam with the geometrical dimensions described in the figure 7 and table 2 . The displacements are completely constrained at the left side. Material parameters are given in table 2 . In order to obtain comparable results, the same time step was employed for the mixed and irreducible approaches. A reference solution was computed employing a very dense finite element mesh (and consequently a very small time step). Calculations are performed with the Multi-physics finite element program KRATOS $[27,28]$, developed at the International Center for Numerical Methods in Engineering (CIMNE). Pre and post-processing is done with GiD, also developed at CIMNE [20]. 


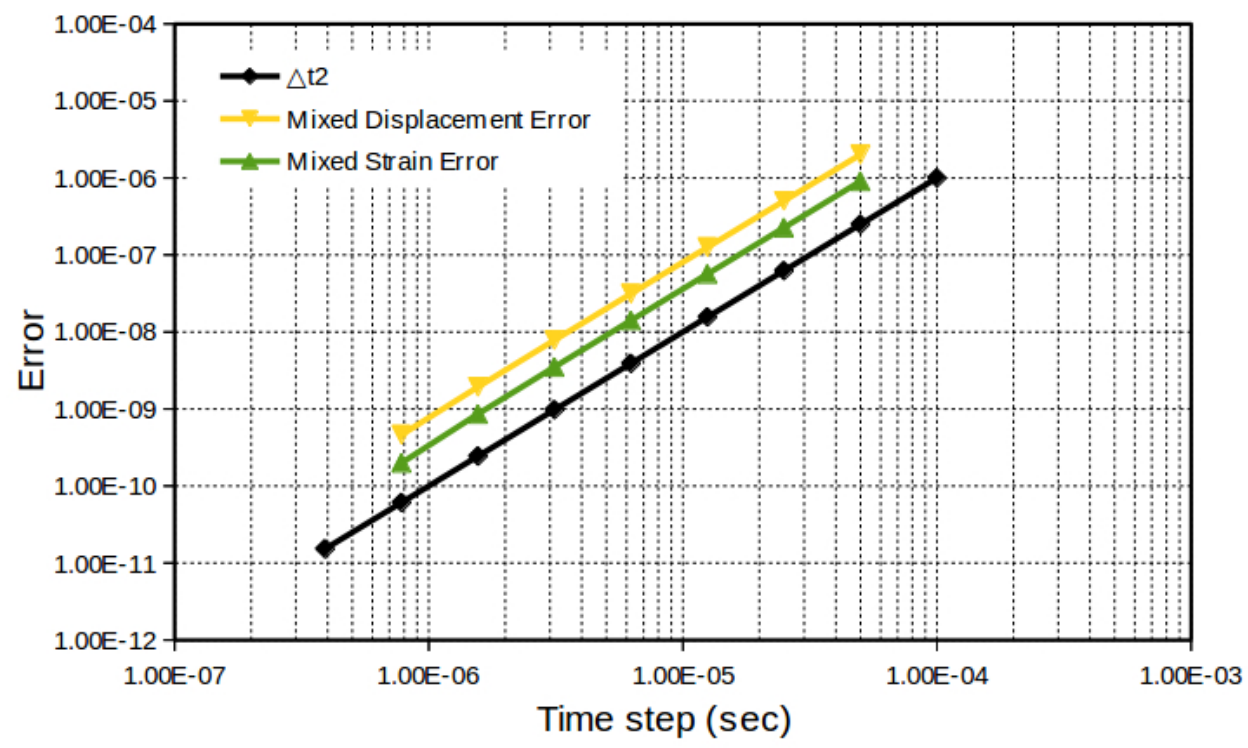

Figure 6: Time error mixed explicit formulation.

\subsubsection{Dynamic cantilever beam. Small deformations case.}

We begin by considering a small deformation case. According to Bernoulli's beam theory, the maximum static deflection $\delta_{\max }^{s t}$ of the cantilever beam under its own weight occurs at its free end (equation 50a). Under the same assumptions the first natural frequency $\omega_{1}$ of the same beam is given in equation $50 \mathrm{~b}$.

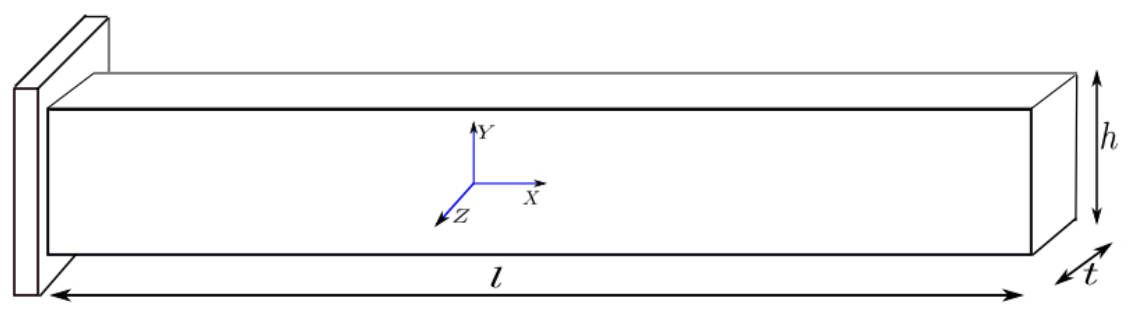

Figure 7: $3 D$ dimension view of cantilever beam.

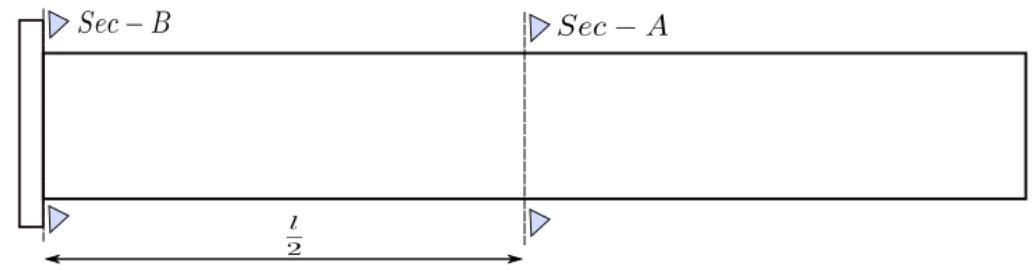

Figure 8: Boundary conditions for small deformations in cantilever beam and section cuts.

$$
\begin{aligned}
\delta_{\max }^{s t} & =u_{\max }=\frac{\rho g A l^{4}}{8 E I} \\
\omega_{1} & =1.875^{2} \sqrt{\frac{E I}{\rho A l^{4}}}
\end{aligned}
$$


For the dynamic case, the maximum dynamic deflection $\delta_{\max }^{\operatorname{din}}$ is expected to be exactly twice the value of the maximum static deflection. For the test we consider three different structured triangle meshes $A$, $B$ and $C$ depicted in the figure 9, 10 and 11 respectively. Details of discretization are shown in the table 3 .

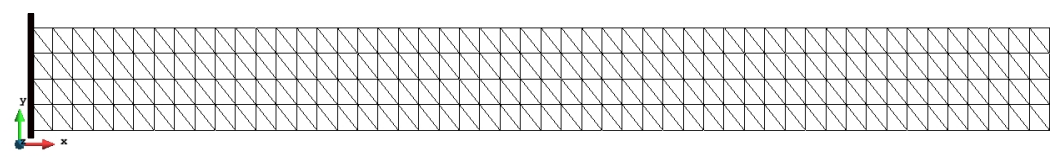

Figure 9: Finite element mesh of cantilever beam model. Mesh $A$

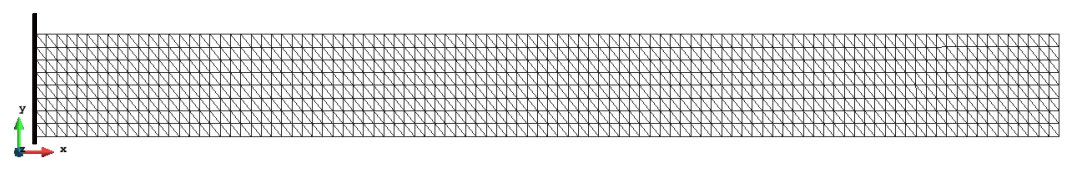

Figure 10: Finite element mesh of cantilever beam model. Mesh $B$

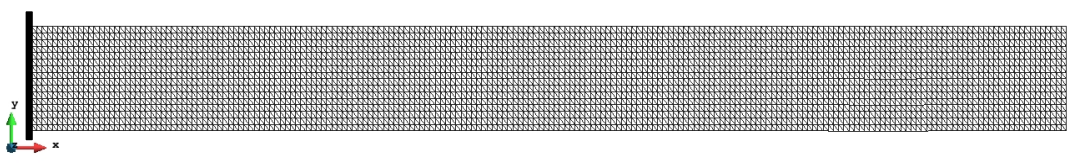

Figure 11: Finite element mesh of cantilever beam model. Mesh $C$

\begin{tabular}{cc}
\hline \hline Material and Geometric Data & Value $(\mathrm{N}-\mathrm{m})$ \\
\hline Young's Modulus $E$ & $2.0 \mathrm{GPa}$ \\
Poisson's ratio $v$ & 0.2 \\
Density $\rho$ & $1000.00 \mathrm{Kg} / \mathrm{m}^{3}$ \\
Length $l$ & $5.00 \mathrm{~m}$ \\
Cross Section $A$ & $0.25 \mathrm{~m}^{2}$ \\
Inertia $I$ & $2.604167 \times 10^{-3} \mathrm{~m}^{4}$ \\
Thickness $t$ & $0.25 \mathrm{~m}$ \\
Gravity $g$ & $9.80665 \mathrm{~m} / \mathrm{s}^{2}$ \\
\hline
\end{tabular}

Table 2: Material and Geometric data for the dynamic analysis of the cantilever beam.

\begin{tabular}{|l|l|l|}
\hline \multicolumn{3}{|c|}{ Discretization Data } \\
\hline Mesh Type & Number of nodes & Number of elements \\
\hline Mesh A & 255 & 400 \\
\hline Mesh B & 909 & 1600 \\
\hline Mesh C & 3417 & 6400 \\
\hline
\end{tabular}

Table 3: Discretization data for Mesh $A, B$ and $C$.

The analytical maximum static deflection $\delta_{\text {max }}^{s t}$ and the first natural frequency $\omega_{1}$ is $18.3875 \mathrm{~mm}$ and $28.705 \mathrm{rad} / \mathrm{s}$ respectively. The corresponding period $T$ of this frequency is $0.219 \mathrm{~s}$. A linear step-bystep dynamic analysis is done using $C D$ for both irreducible and mixed formulation. The results of the analysis are depicted in figures 12,13 and 14. They correspond to absolute values of vertical displacement 
(displacement Y direction) evolution at free end (point Q in figure 8). As expected, for values of $\tau_{\varepsilon}$ in the typical range 0.1-0.5, at any given mesh resolution, the mixed formulation is slightly more accurate than the irreducible one. This is true both in terms of phase accuracy and in terms of stiffness. Interestingly, depending on the value of the stabilization parameter the structure can be either too flexible or too stiff compared to the analytical solution, which is not unexpected since a mixed-formulation may converge from either side. As expected, as the mesh is refined, all formulations converge to the reference solution.

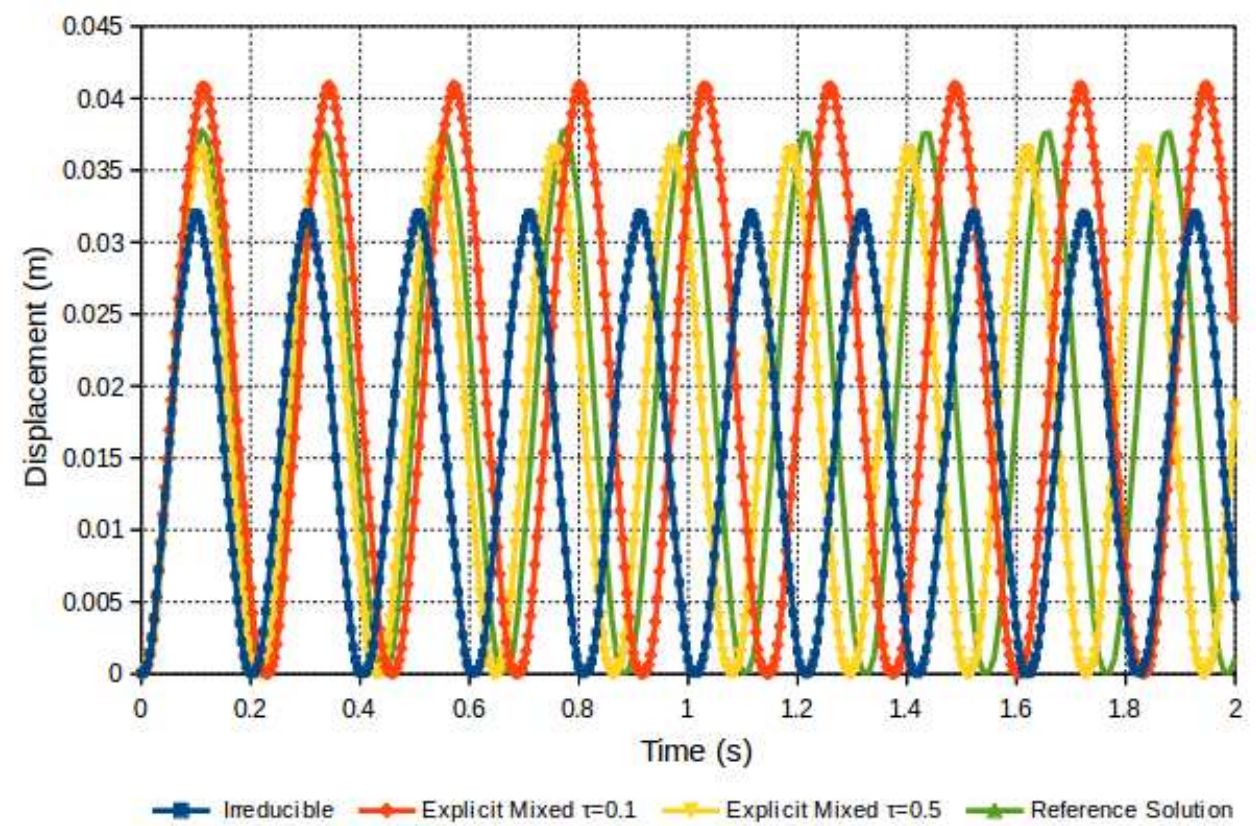

Figure 12: Step-by-step dynamic analysis using both irreducible and mixed explicit formulation for mesh A.

As a remark we observe that as shown in previous works[7, 8, 15], where the static case was explored, the explicit mixed formulation is always (independently on the $\tau_{\varepsilon}$ ) more accurate than the irreducible formulation on a given mesh.

\subsubsection{Dynamic cantilever beam. Large deformations case.}

As a second example, we consider the same settings and increase by a factor 100 the applied load (100 times higher gravity load), thus triggering a large deformation response. The results shown in figure 15 , 16 and 17 depicts the displacement of the tip (intended as the center point of the rightmost face) for the three meshes identified as $A, B$ and $C$.

As it can be expected convergence is guaranteed, even for large deformations cases using a mixed-explicit Total Lagrangian approach. It is also interesting to compare the irreducible and mixed formulations Green-Lagrange strain distributions over two different cross sections as shown in figure 8 .

The strain distribution in the middle-right section and at the constraint is shown in figures 18 and 19 for mesh $A, 20$ and 21 for mesh $B$ and 22 and 23 for mesh $C$.

As the images suggest, and in accordance to the theory, the strain spatial convergence is faster for the mixed formulation than for the irreducible one. Note that already for the structured non-symmetric coarse mesh (mesh A), the distribution of strains in the section gives sensibly better results with respect to the irreducible one. The strain distribution also appears to converge faster to the reference result as the mesh is refined.

Applying the mixed formulation, in pictures 24, 25 and 26 we observe how the imposed load excites the first, second and third modes of the beam. As expected, if a finer discretization is used more vibration 


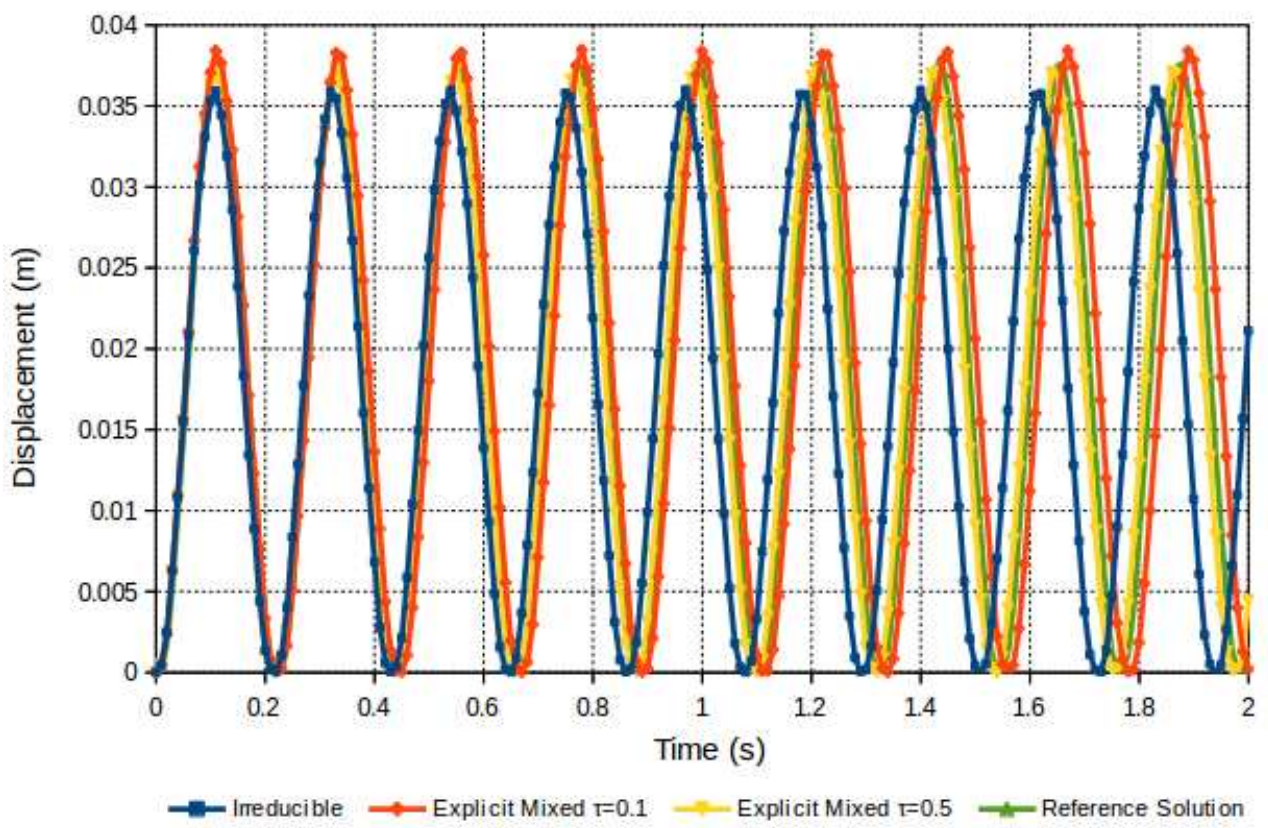

Figure 13: Step-by-step dynamic analysis using both irreducible and mixed explicit formulation for mesh B.

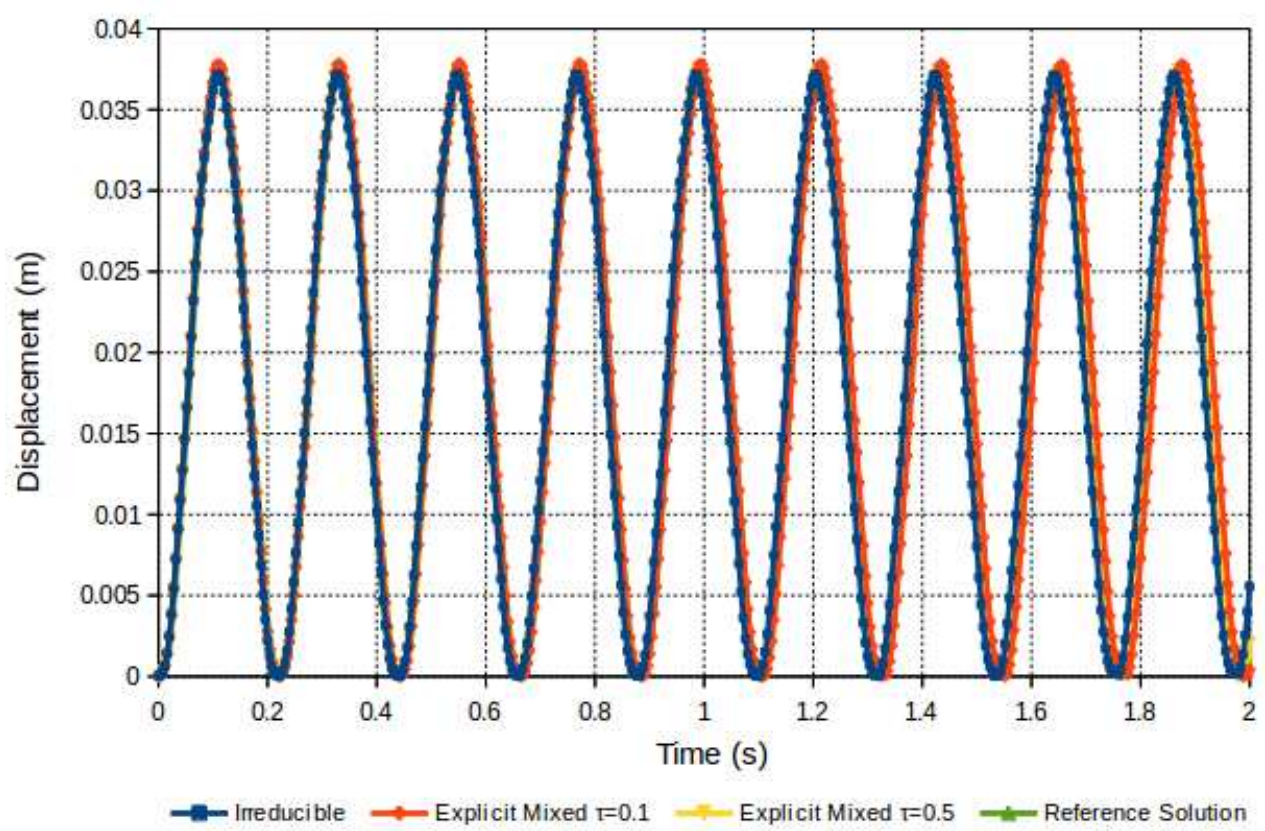

Figure 14: Step-by-step dynamic analysis using both irreducible and mixed explicit formulation for mesh C.

modes appear in the analysis.

Since the stability analysis developed is only valid for the linear case, we experimentally increased the time step used in the analysis until the method failed, so to approximate the largest possible time step. 


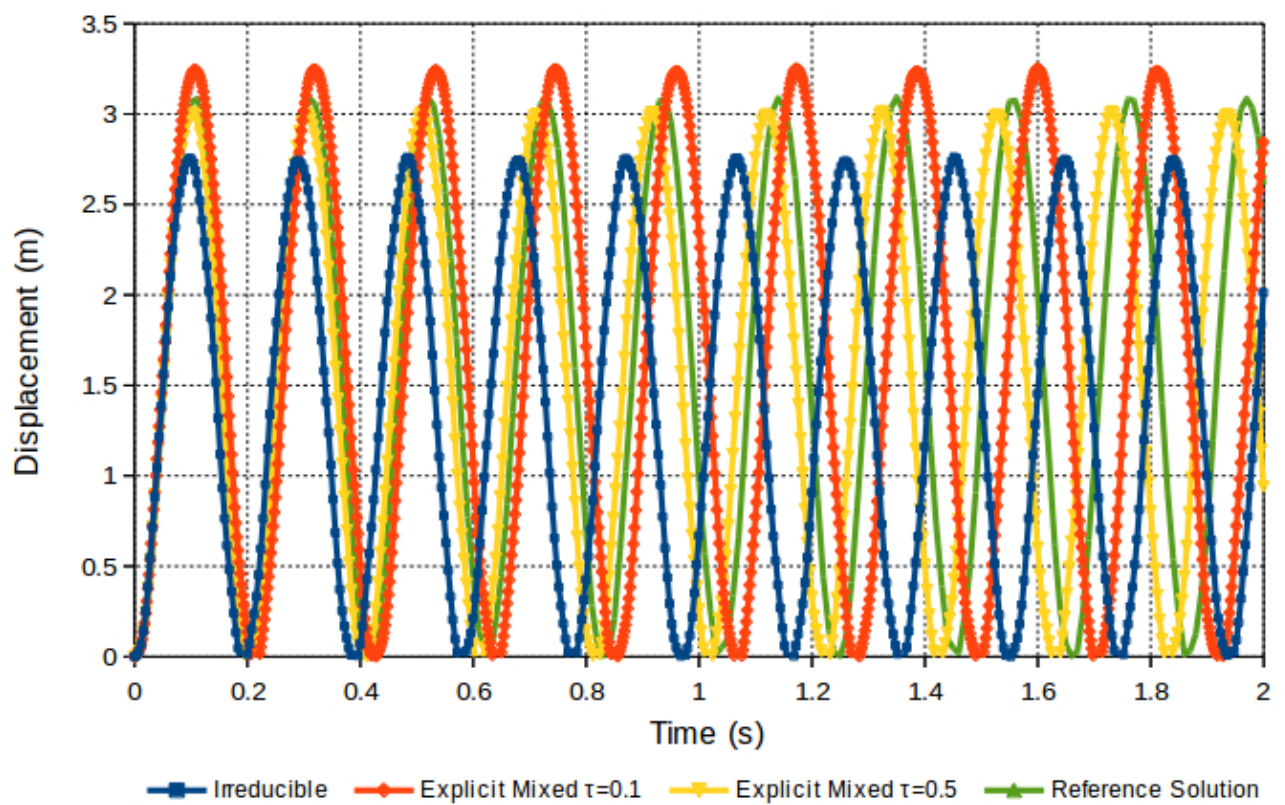

Figure 15: Step-by-step dynamic analysis using both irreducible and mixed CD scheme for mesh A.

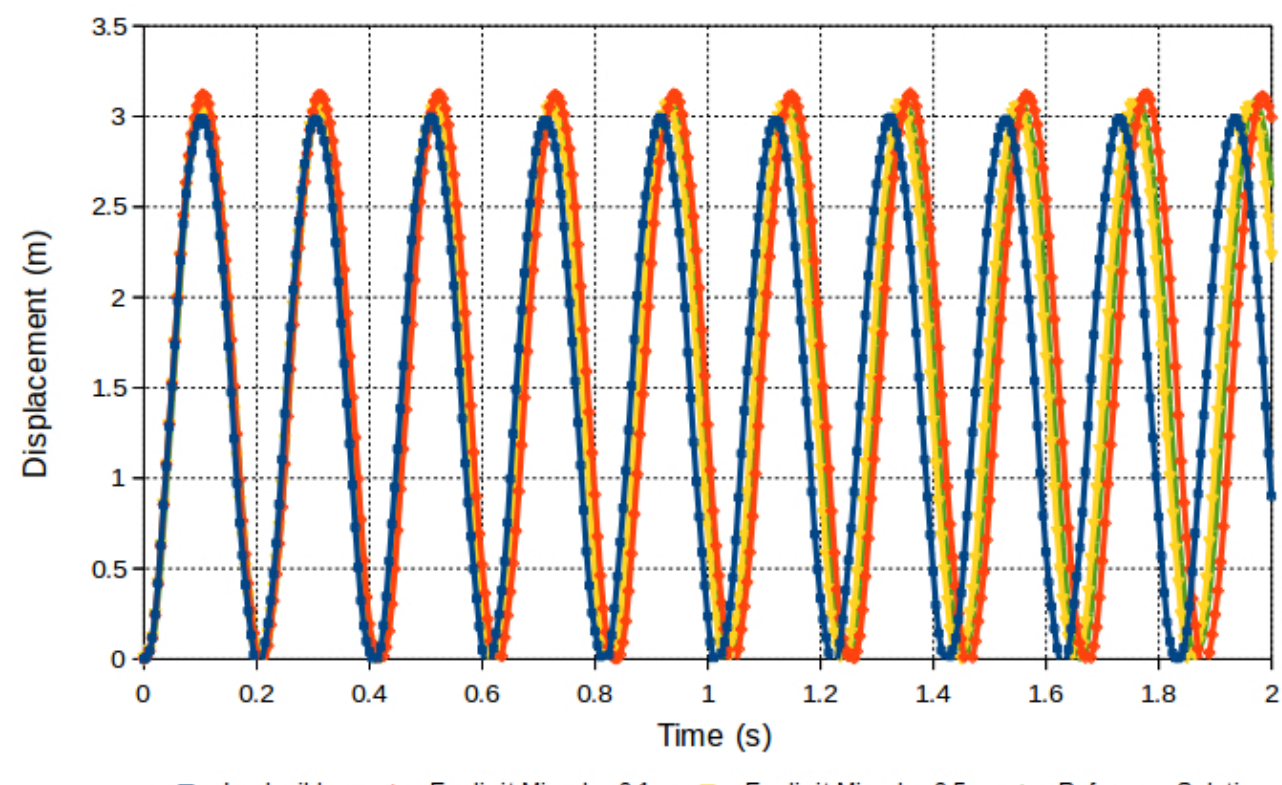

Figure 16: Step-by-step dynamic analysis using both irreducible and mixed CD scheme for mesh B.

The results are shown in table 4 together with the time step for one-dimensional mixed and irreducible case. We approximate the length of the triangular element $l_{e}$ as the half-root square of the quadrilateral area closed by the triangle, i.e; $l_{e}=\frac{1}{2} \sqrt{b h}$. As we can note, even for large deformation case the time step used in the mixed-explicit formulation is larger than irreducible one, closely following the predictions in figure 5. Specifically we numerically verify that the stable time step for this example is at least a factor of 1.43 larger for the mixed formulation than for the original irreducible one, which represents a very important performance gain for the proposed formulation. 


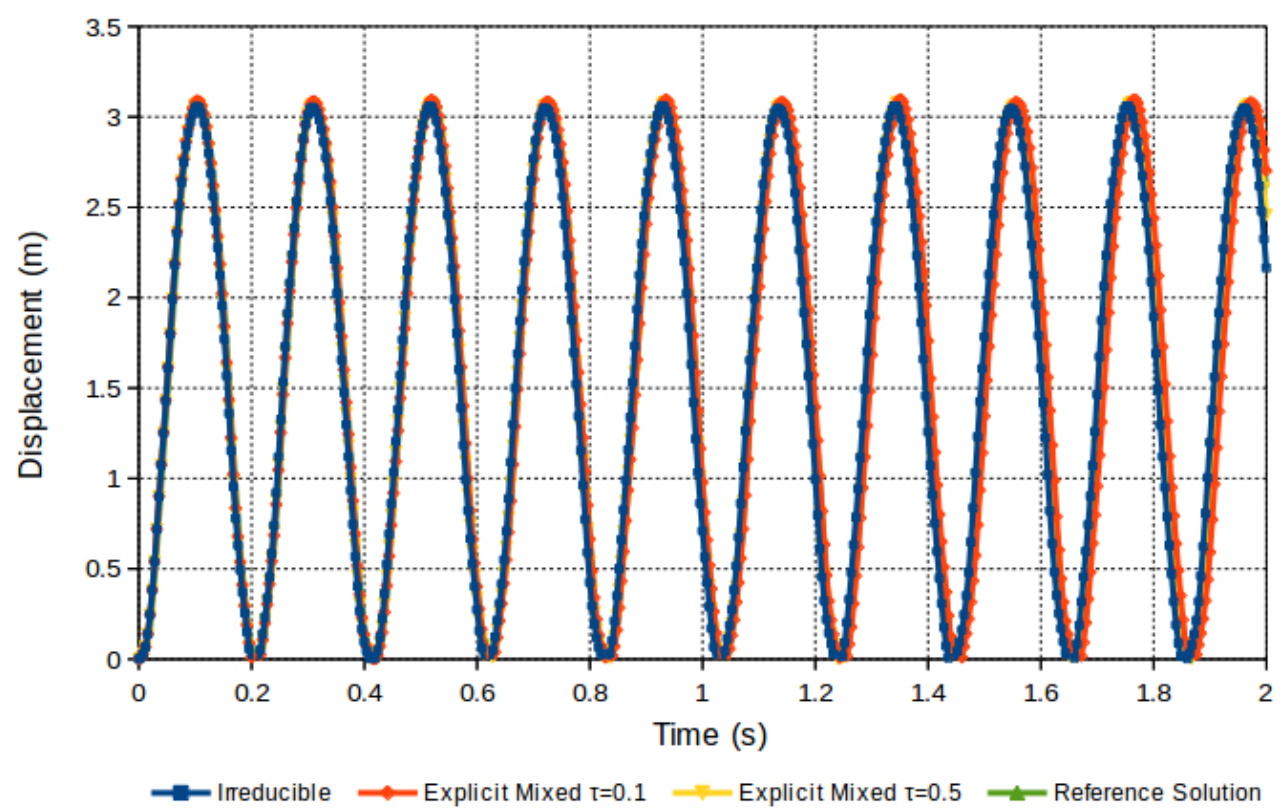

Figure 17: Step-by-step dynamic analysis using both irreducible and mixed CD scheme for mesh C.

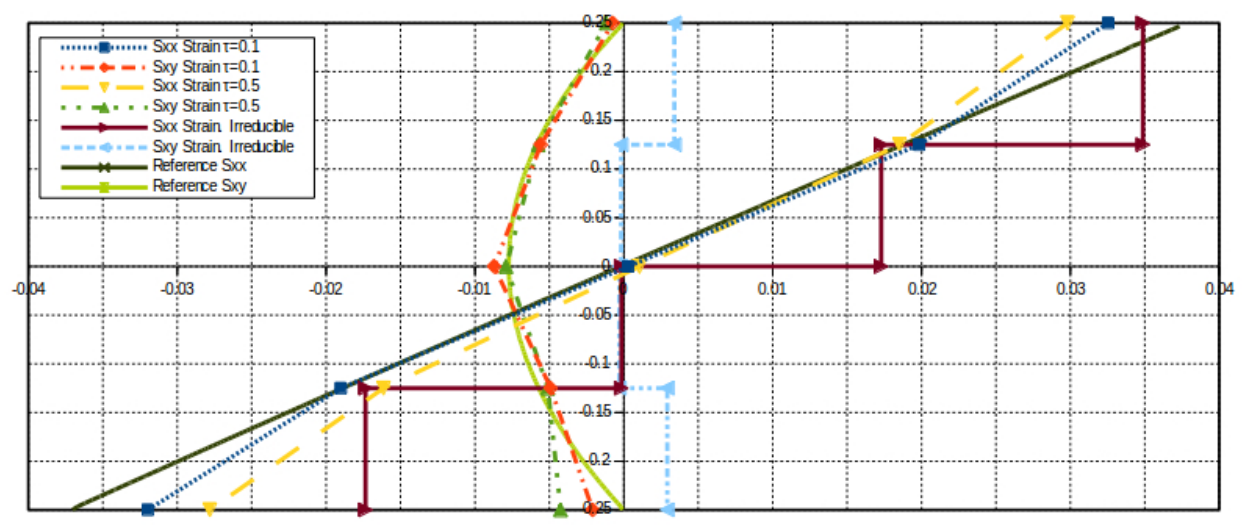

Figure 18: Section A normal shear strain distribution at maximum displacement for mesh $A$.

\begin{tabular}{|l|l|l||l||l||l|}
\hline \multicolumn{9}{|c|}{ Critical $\Delta t^{\text {max }}$ computed } \\
\hline Mesh Type & $\Delta t^{\text {max }}$ Irreducible 1D & $\Delta t^{\text {max }}$ Irreducible & $\Delta t^{\text {max }}$ Mixed 1D & $\Delta t^{\text {max }}$ Mixed & factor \\
\hline Mesh A & $1.76 \mathrm{e}-04 s$ & $5.30 \mathrm{e}-5 s$ & $2.38 \mathrm{e}-04 s$ & $7.585 \mathrm{e}-5 s$ & 1.43 \\
\hline Mesh B & $8.83 \mathrm{e}-05 s$ & $2.55 \mathrm{e}-5 s$ & $1.19 \mathrm{e}-04 s$ & $3.795 \mathrm{e}-5 s$ & 1.49 \\
\hline Mesh C & $4.41 \mathrm{e}-05 s$ & $1.07 \mathrm{e}-5 s$ & $5.95 \mathrm{e}-05 s$ & $1.80 \mathrm{e}-05 s$ & 1.68 \\
\hline
\end{tabular}

Table 4: Maximum $\Delta t^{\max }$ for analysis in irreducible and mixed formulation.

\section{Conclusion}

This paper presents the formulation of a stable, OSS based, mixed explicit strain/displacement formulation for the solution of linear and non-linear problems is solid mechanics. In the work we describe a simple algorithm allowing the explicit time integration of such mixed formulation. The resulting explicit scheme retains second order accuracy in time and has a favourable stability estimate when compared to the ir- 


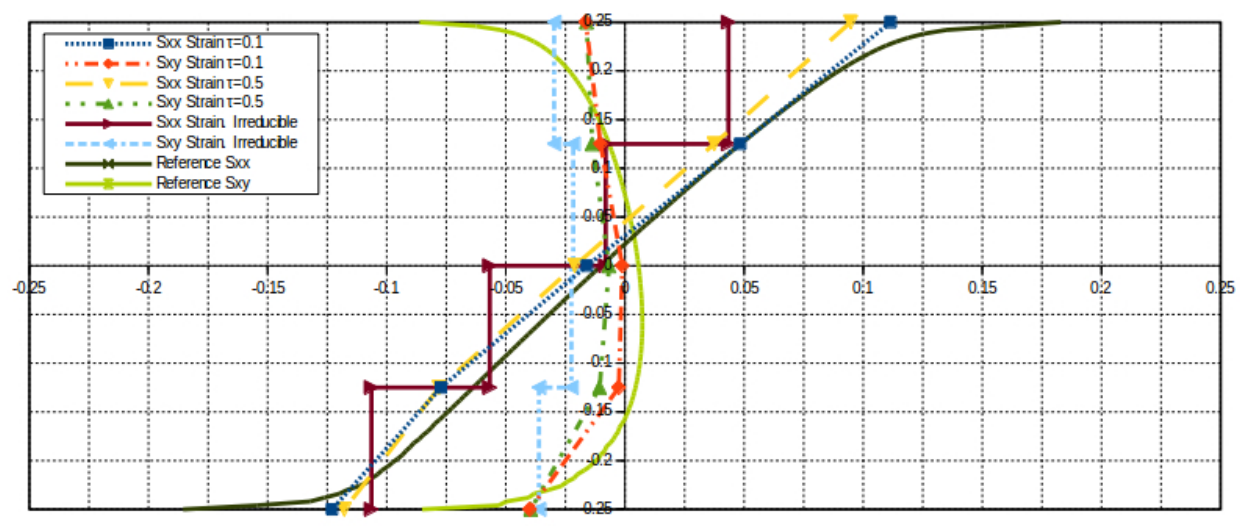

Figure 19: Section B normal shear strain distribution at maximum displacement for mesh $A$.

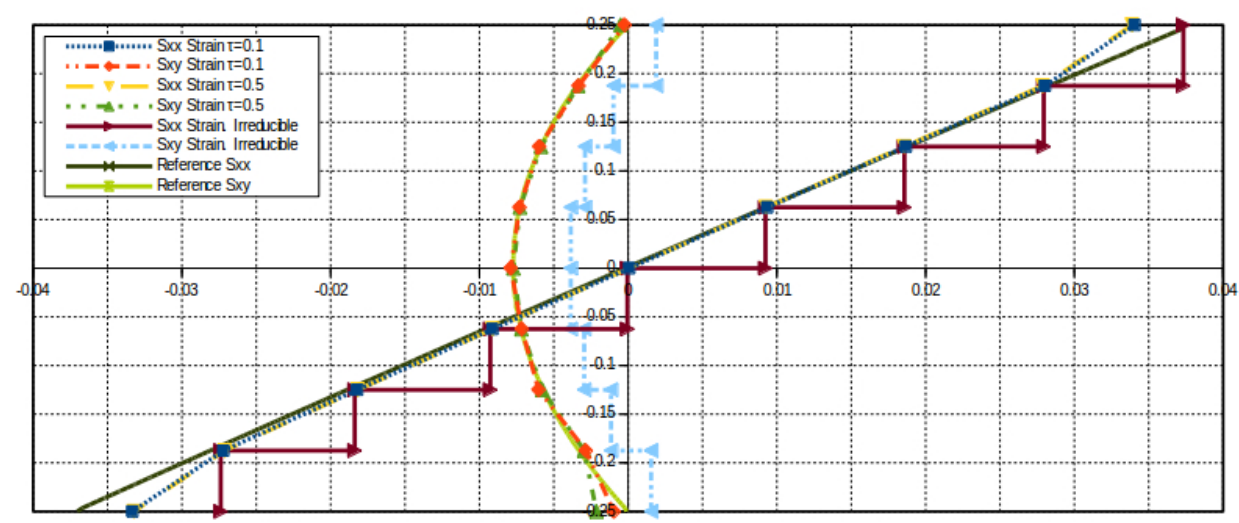

Figure 20: Section A normal shear strain distribution at maximum displacement for mesh $B$.

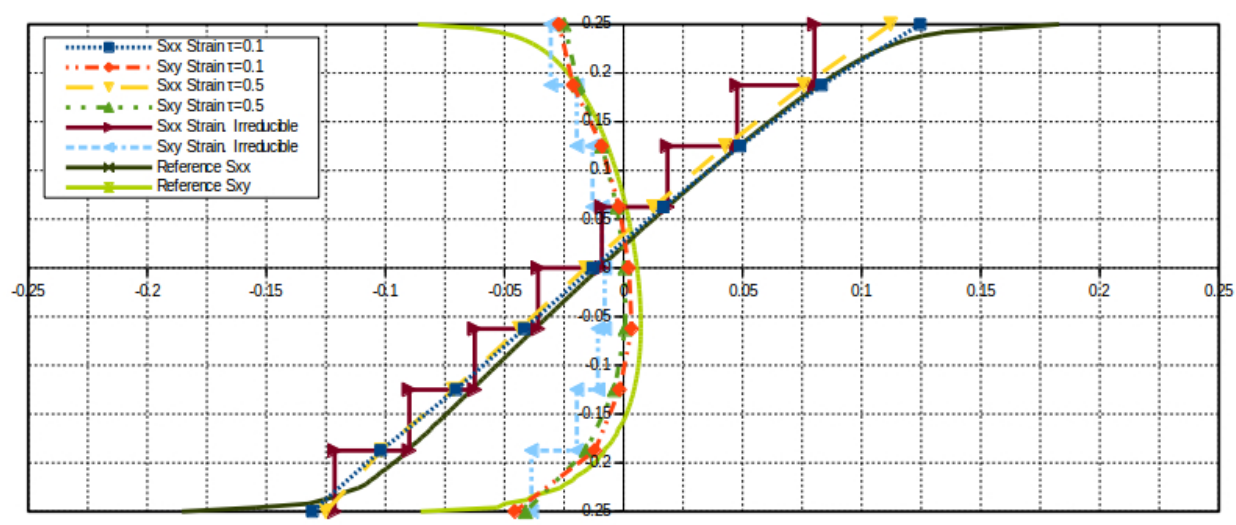

Figure 21: Section B normal shear strain distribution at maximum displacement for mesh $B$.

reducible formulation. The numerical results also show how the results obtained compare favourably in terms of accuracy with the corresponding irreducible formulations at any mesh resolution. The advantage is particularly evident in the strain predictions. 


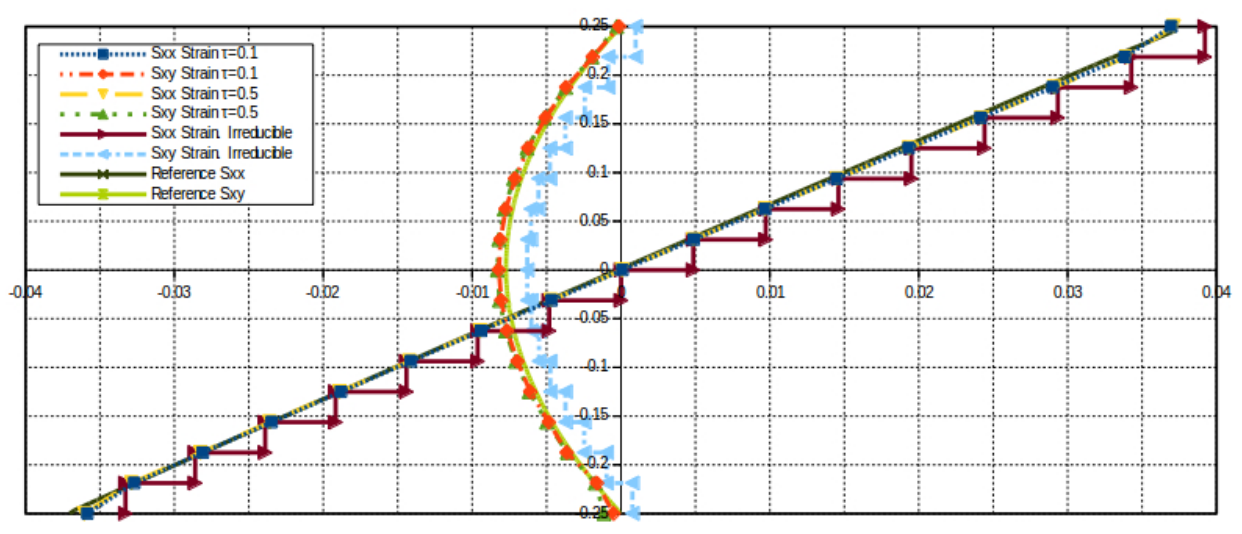

Figure 22: Section A normal shear strain distribution at maximum displacement for mesh $C$.

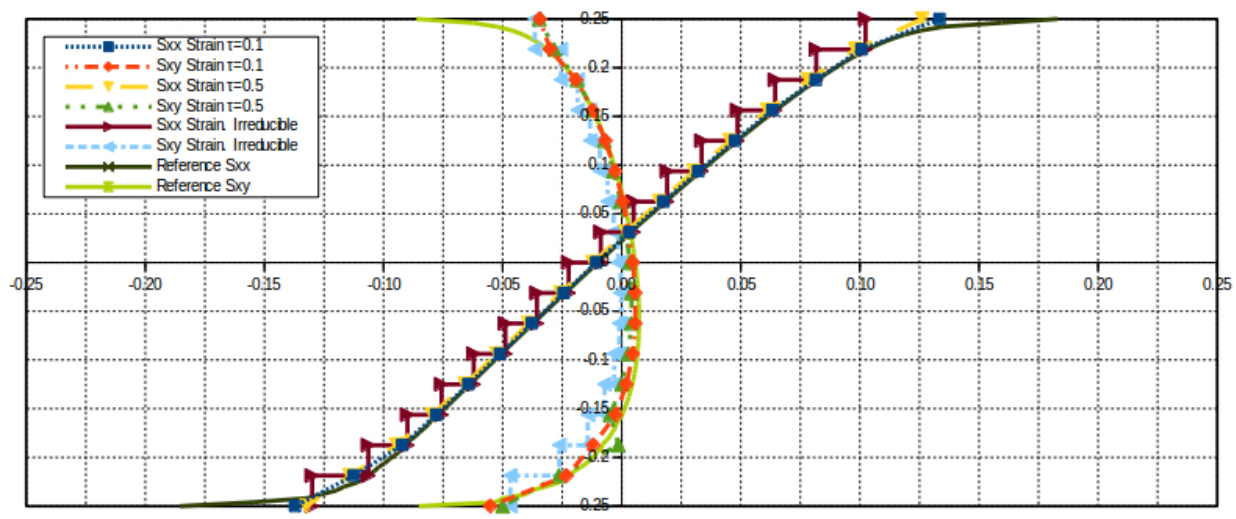

Figure 23: Section B normal shear strain distribution at maximum displacement for mesh $C$.

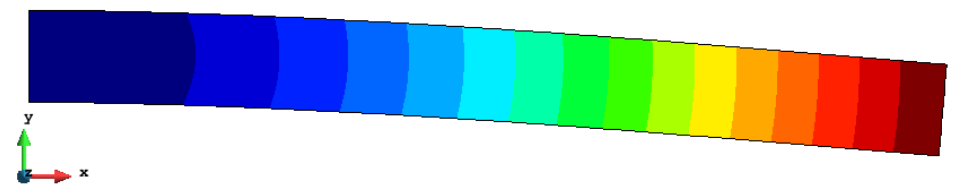

Figure 24: First vibration mode of the cantilever beam in mesh $C$.

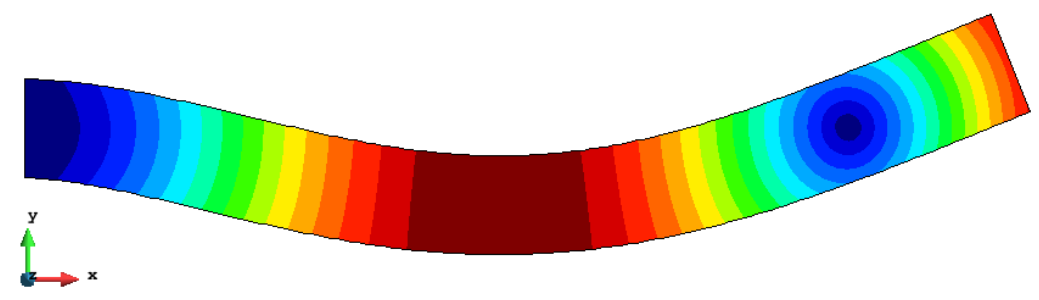

Figure 25: Second vibration mode of the cantilever beam in mesh $C$.

\section{Acknowledgements}

Nelson Lafontaine thanks to MAEC-AECID scholarships for the financial support given. Funding from the Seventh Framework Programme (FP7/2007-2013) of the ERC under grant agreement n 611636 


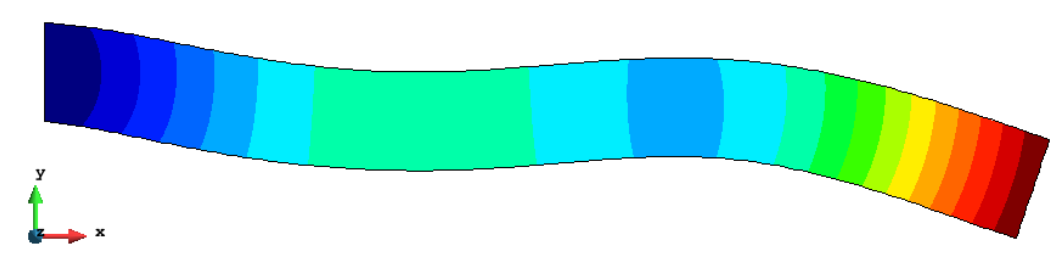

Figure 26: Third vibration mode of the cantilever beam in mesh $C$.

(NUMEXAS) has helped the development of this project. The authors also wish to thank Mr. Pablo Becker for his help in the revision process.

\section{References}

[1] Neto, E. A.de Souza and Pires, F. M. Andrade and Owen, D. R. J. F-bar-based linear triangles and tetrahedra for finite strain analysis of nearly incompressible solids. part i: formulation and benchmarking. International Journal for Numerical Methods in Engineering, 62(3):353-383, 2005.

[2] Antonio J. Gil and Chun Hean Lee and Javier Bonet and Miquel Aguirre. A stabilised petrov-galerkin formulation for linear tetrahedral elements in compressible, nearly incompressible and truly incompressible fast dynamics. Computer Methods in Applied Mechanics and Engineering, 276(0):659-690, 2014

[3] David S. Malkus and Thomas J.R. Hughes. Mixed finite element methods — reduced and selective integration techniques: A unification of concepts. Computer Methods in Applied Mechanics and Engineering, 15(1):63 - 81, 1978.

[4] J.C. Simo and R.L. Taylor and K.S. Pister. Variational and projection methods for the volume constraint in finite deformation elasto-plasticity. Computer Methods in Applied Mechanics and Engineering, 51(1-3):177 - 208, 1985.

[5] Taylor, Robert L. A mixed-enhanced formulation tetrahedral finite elements. International Journal for Numerical Methods in Engineering, 47(1-3):205-227, 2000.

[6] G. Scovazzi. Lagrangian shock hydrodynamics on tetrahedral meshes: A stable and accurate variational multiscale approach. Journal of Computational Physics, 231(24):8029 - 8069, 2012.

[7] M. Chiumenti M. Cervera and R. Codina. Mixed stabilized finite element methods in nonlinear solid mechanics: Part i: Formulation. Computer Methods in Applied Mechanics and Engineering, 199(37-40):2559-2570, 2010.

[8] M. Cervera, M. Chiumenti, and R. Codina. Mixed stabilized finite element methods in nonlinear solid mechanics: Part ii: Strain localization. Computer Methods in Applied Mechanics and Engineering, $199(37-40): 2571-2589,2010$

[9] Ramon Codina. Stabilized finite element approximation of transient incompressible flows using orthogonal subscales. Computer Methods in Applied Mechanics and Engineering, 191(39-40):4295 - 4321, 2002.

[10] Jean Donea and Antonio Huerta. Finite Element Methods for Flow Problems. Wiley, 1 edition, June 2003.

[11] Thomas J.R. Hughes. Multiscale phenomena: Green's functions, the dirichlet-to-neumann formulation, subgrid scale models, bubbles and the origins of stabilized methods. Computer Methods in Applied Mechanics and Engineering, 127(1-4):387 - 401, 1995.

[12] E. Oñate, A. Valls, and J. García. Fic/fem formulation with matrix stabilizing terms for incompressible flows at low and high reynolds numbers. Computational Mechanics, 38(4-5):440-455, 2006. 
[13] Thomas J. R. Hughes, Guglielmo Scovazzi, and Leopoldo P. Franca. Multiscale and Stabilized Methods. John Wiley \& Sons, Ltd, 2004.

[14] Thomas J.R. Hughes and Gonzalo R. Feijóo and Luca Mazzei and Jean-Baptiste Quincy. The variational multiscale method - a paradigm for computational mechanics. Computer Methods in Applied Mechanics and Engineering, 166(1-2):3 - 24, 1998. Advances in Stabilized Methods in Computational Mechanics.

[15] M. Cervera, M. Chiumenti, and R. Codina. Mesh objective modeling of cracks using continuous linear strain and displacement interpolations. International Journal for Numerical Methods in Engineering, 87(10):962-987, 2011.

[16] T. Belytschko, W. K. Liu, and B. Moran. Nonlinear Finite Elements for Continua and Structures. John Wiley and Sons, Ltd., New York, 2000.

[17] Har, Jason and Fulton, Robert E. A parallel finite element procedure for contact-impact problems. Engineering with Computers, 19:67-84, 2003. 10.1007/s00366-003-0252-4.

[18] M. Cervera and M. Chiumenti. Mesh objective tensile cracking via a local continuum damage model and a crack tracking technique. Computer Methods in Applied Mechanics and Engineering, 196(1-3):304-320, 2006.

[19] T.A. Laursen. Computational Contact and Impact Mechanics: Fundamentals of Modeling Interfacial Phenomena in Nonlinear Finite Element Analysis. Engineering Online Library. Springer, 2003.

[20] GiD. The personal pre and post processor. 2009.

[21] F. Brezzi, M. Fortin, D. Marini. Mixed finite element methods. Springer, 1991.

[22] J. Blasco R. Codina. A finite element method for the stokes problem allowing equal velocity-pressure interpolations. Comput. Meth Appl Mech Eng, 143:373-391, 1997.

[23] R. Codina. Stabilization of incompresssibility and convection through orthogonal sub-scales in finite elements methods. Comput. Meth Appl Mech Eng, 190:1579-1599, 2000.

[24] Ramon Codina. Analysis of a stabilized finite element approximation of the oseen equations using orthogonal subscales. Appl. Numer. Math., 58(3):264-283, mar 2008.

[25] A. Larese, R. Rossi, E. Oñate, and S.R. Idelsohn. A coupled pfem-eulerian approach for the solution of porous fsi problems. Computational Mechanics, 50(6):805-819, 2012.

[26] A. Larese, R. Rossi, E. Oñate, M. Toledo, R. Morán, and H. Campos. Numerical and experimental study of overtopping and failure of rockfill dams. International Journal of Geomechanics, 0(0):04014060, 0 .

[27] P. Dadvand, R. Rossi, M. Gil, X. Martorell, J. Cotela, E. Juanpere, S.R. Idelsohn, and E. Oñate. Migration of a generic multi-physics framework to hpc environments. Computers $\&$ Fluids, 80(0):301 - 309, 2013.

[28] Pooyan Dadvand and Riccardo Rossi and Eugenio Oñate. An object-oriented environment for developing finite element codes for multi-disciplinary applications. Archives of Computational Methods in Engineering, 17(3):253-297, 2010.

[29] O. C. Zienkiewicz, R. L. Taylor, and J. Z. Zhu. The Finite Element Method: Its Basis and Fundamentals, Sixth Edition. Butterworth-Heinemann, 6 edition, May 2005.

[30] Zienkiewicz, O. C. and Rojek, J. and Taylor, R. L. and Pastor, M. Triangles and tetrahedra in explicit dynamic codes for solids. International Journal for Numerical Methods in Engineering, 43(3):565-583, 1998.

[31] Zienkiewicz, O. C. and Taylor, R. L. and Baynham, J.AW. Mixed and irreducible formulations in finite element analysis, in hybrid and mixed finite element methods. S.N Atlury, R.H. Gallagher and O. C Zienkiewicz Eds., Wiley, 1983. 
[32] Douglas N. Arnold. Mixed finite element methods for elliptic problems. Computer Methods in Applied Mechanics and Engineering, 82(1-3):281 - 300, 1990. Proceedings of the Workshop on Reliability in Computational Mechanics.

[33] Douglas N. Arnold and Ragnar Winther. Mixed finite elements for elasticity in the stressdisplacement formulation. Contemp. Math.,, 239:33-42, 2003. n Z. Chen, R. Glowinski, and K. Li, editors, Current trends in scientific computing (Xi'an, 2002).

[34] Arnold, Douglas N. and Winther, Ragnar. Mixed finite elements for elasticity. Numerische Mathematik, 92(3):401-419, 2002.

[35] M. Pastor and T. Li and X. Liu and O.C. Zienkiewicz. Stabilized low-order finite elements for failure and localization problems in undrained soils and foundations. Computer Methods in Applied Mechanics and Engineering, 174(1-2):219 - 234, 1999.

[36] Babuska, Ivo. Error-bounds for finite element method. Numerische Mathematik, 16(4):322-333, 1971.

[37] C. Agelet de Saracibar and M. Chiumenti and Q. Valverde and M. Cervera. On the orthogonal subgrid scale pressure stabilization of finite deformation $\{\mathrm{J} 2\}$ plasticity. Computer Methods in Applied Mechanics and Engineering, 195(9-12):1224 - 1251, 2006.

[38] Ottmar Klaas and Antoinette Maniatty and Mark S. Shephard. A stabilized mixed finite element method for finite elasticity.: Formulation for linear displacement and pressure interpolation. Computer Methods in Applied Mechanics and Engineering, 180(1-2):65 - 79, 1999.

[39] Oñate, Eugenio and Rojek, Jerzy and Taylor, Robert L. and Zienkiewicz, Olgierd C. Finite calculus formulation for incompressible solids using linear triangles and tetrahedra. International Journal for Numerical Methods in Engineering, 59(11):1473-1500, 2004.

[40] Antoinette M. Maniatty and Yong Liu and Ottmar Klaas and Mark S. Shephard. Stabilized finite element method for viscoplastic flow: formulation and a simple progressive solution strategy. Computer Methods in Applied Mechanics and Engineering, 190(35-36):4609 - 4625, 2001.

[41] Cervera, M. and Chiumenti, M. and Agelet de Saracibar, C. Softening, localization and stabilization: capture of discontinuous solutions in j2 plasticity. International Journal for Numerical and Analytical Methods in Geomechanics, 28(5):373-393, 2004.

[42] M. Chiumenti and Q. Valverde and C. Agelet de Saracibar and M. Cervera. A stabilized formulation for incompressible elasticity using linear displacement and pressure interpolations. Computer Methods in Applied Mechanics and Engineering, 191(46):5253 - 5264, 2002.

[43] M Chiumenti and Q Valverde and C Agelet de Saracibar and M Cervera. A stabilized formulation for incompressible plasticity using linear triangles and tetrahedra. International Journal of Plasticity, 20(8-9):1487 - 1504, 2004.

[44] M. Cervera and M. Chiumenti and C. Agelet de Saracibar. Shear band localization via local \{J2\} continuum damage mechanics. Computer Methods in Applied Mechanics and Engineering, 193(9-11):849 - 880, 2004.

[45] Baiges, Joan and Codina, Ramon. A variational multiscale method with subscales on the element boundaries for the helmholtz equation. International Journal for Numerical Methods in Engineering, 93(6):664-684, 2013.

[46] Badia, S. and Codina, R. Unified stabilized finite element formulations for the stokes and the darcy problems. SIAM Journal on Numerical Analysis, 47(3):1971-2000, 2009. 